\title{
Decreasing arousal modulates perceptual decision-making
}

${ }^{1}$ Department of Psychology, University of Cambridge, Cambridge, United Kingdom

${ }^{2}$ Department of Clinical Neurosciences, University of Cambridge, Cambridge, United Kingdom

${ }^{3}$ School of Psychology, Victoria University of Wellington, Wellington, New Zealand 8

*Corresponding authors: sri34@cam.ac.uk, tb419@cam.ac.uk

\section{ABSTRACT}

The ability to make decisions based on external information, prior knowledge, evidence and context, is a crucial aspect of cognition and may determine the success and survival of an organism. Despite extensive work on decision making mechanisms and models, understanding the effects of arousal on its neural and cognitive processes remain limited. Here we characterise behavioural and neural dynamics of perceptual decision making in awake and low alertness periods in humans, and characterise the compensatory mechanisms as arousal decreases. Well-rested human participants, fluctuating between full wakefulness and low alertness, performed an auditory tone-localisation task and its behavioural dynamics was quantified with psychophysics, signal detection theory and drift-diffusion modelling, revealing slower reaction times, inattention to the left side of space, and a lower rate of evidence accumulation in periods of low alertness. Unconstrained multivariate pattern analysis (decoding) showed a $\sim 260 \mathrm{~ms}$ delayed onset driven by low alertness of the neural signatures differentiating between left and right decision, with a spatial reconfiguration from centro-parietal to lateral frontal regions $160-300 \mathrm{~ms}$. To understand the neural compensatory mechanisms with decreasing arousal, we connected the evidence-accumulation behavioural parameter to the neural activity, showing in the early period $(150-300 \mathrm{~ms})$ a shift in the associated patterns from bilateral inferior frontal gyri in awake to right dorsolateral in low alertness. The second period of evidence accumulation shows posterior involvement in awake and, due to the delayed processing during drowsiness, a wide frontopolar, dorsolateral prefrontal cortices and right parietal during decreased arousal. The change in the neurobehavioural dynamics for both perceptual encoding and central accumulation cognitive processes define a clear reconfiguration of the brain networks' regions and dynamics needed for the implementation of decision making in both early perceptual and later central processes, revealing compensatory mechanisms of resilience of cognition when challenged by decreased arousal. 


\section{INTRODUCTION}

The question of how decisions are made has shaped the world's systems of government, justice and social order (1). From a cognitive neuroscience perspective, the modelling of psychological and neural factors allows for the understanding of decision making via its underlying brain mechanisms, depending both from the specific constraints of the information provided from the external world and the internal state of the corresponding system. Studies on how the brain implements simple decisions have revealed several neurocognitive processes at the perceptual, central integration and motor implementation levels (2-4), but the modulatory effects of the internal milieu, homeostasis, arousal, alertness, and circadian influences on such processes have received less attention $(5,6)$. Specifically, low alertness fluctuations on decision processes have been tackled by sleep deprivation and brain injury studies but hardly by normal variations in the stability of wakefulness (7). How does the brain compensate for the detrimental effects of decreasing arousal while making an optimal decision?

Perceptual decision making is successfully studied under the sequential sampling models $(8,9)$, and referred to as a set of processes involving the processing of sensory information to make a behavioural choice from a set of available options (10). The various elements of this process (Figure s1) are: a) Perceptual stage: a sensory system that transforms physical stimulus intensities for the perceptual systems to deliver decision information; b) Central integration stage: a decision system that integrates and accumulates such decision-information variables and makes an optimal choice based on relative evidence; and c) Motor stage: a motor system that implements the appropriate motor plan and subsequent action. According to sequential sampling models, accurate perceptual decisions separate the noise from the signal by repeatedly sampling and integrating evidence until there is enough in favour of one of the decision choices. The preferred concept to understand and develop hypotheses is called decision variable, and it is an accumulation of priors, evidence, internal milieu and value into a quantity that is interpreted by the decision rule to produce a choice (9). This study aims to investigate how alertness modulates the decision variable in spatial auditory perception.

The neural underpinnings of perceptual decisions have been explored both in model-free and model-constrained manners in neuroimaging studies in rodents, monkeys and human observers. Signals from domain general regions such as the right insula, posterior left SFS/DLPFC, left TPJ, posterior parietal cortex (LIP) were related to processes of perceptual and central evidence accumulation (11-16). The above studies provide a general overview on the different regions of the brain involved in processes related to decision-making. To explore the question of how alertness modulates these decision-making related processes, we need to first define the specific aspect of wakefulness to be used as the experimental manipulation $(17,18)$. Alertness related modulations can 
be classed into 'tonic' fluctuations that span multiple trials and time-periods, and 'phasic' moment to moment modulations in arousal produced in response to an ongoing task. A few recent studies have shown, in humans and mice, that arousal, measured by brain-stem systems (pupil response), modulates individual decision making in moment to moment fluctuations of phasic arousal $(19,20)$. Further to this, van Kempen and collaborators (21) showed that lower tonic and higher phasic arousal, as defined by the pupil measurements, predicted shorter reaction times and was associated with a centroparietal positivity in EEG space. However, these studies have used pupil responses; which is a physiological marker indirectly linked to neural arousal systems, and further, can also be influenced by other factors like visual contrast, spatial frequencies of the stimulus etc. (22). In addition to this, the above mentioned studies have not measured influences of tonic fluctuations in decreased arousal, which is difficult for pupillometry or eye tracker measures. Hence, we require a study that can directly investigate the effect of intrinsic low alertness (tonic) fluctuations on decision-making.

Before undertaking such an investigation, we need to resolve the problem of measuring arousal relatively independently and directly from the brain. For this, we use subclassification of stages of alertness pioneered by Hori and collaborators (23). This manual method of scoring is based on spectral power as a continuous measure and visually defined grapho-elements in the transition to sleep. We took a computational approach to this problem, validated and automated this procedure to avoid human error, and further cross-validated it to show robust outcomes (24) across different EEG datasets $(64,128$ channel) with participants performing a variety of tasks (semantic decision-making, auditory masking). Recently this method was successfully applied to explore the effect of alertness on cognitive control (18).

This sets up the stage for the primary questions that will be analysed in this study: a) In terms of behaviour, how does a perceptual decision making task get affected by changing levels of tonic alertness. b) How are such modulations in behaviour connected to different elements of the decision making process i.e. perceptual, central, motor stage (Figure s1) and how are these stages modulated by alertness. c) How are the specific temporal periods and spatial regions implementing the different elements of the decision making process modulated by alertness. d) Finally, how does the brain, when compromised by lower levels of alertness, adapt/reconfigure to perform the decision.

To answer the above questions, we decided to use an experiment (auditory spatial localisation task) with a known behavioural asymmetry (25) and understand how neurobehavioural decision-making mechanisms are modulated by fluctuations in tonic alertness. First, we use a combination of multi-level modelling, psychophysics and signal detection theory to show how behaviour is modulated by variations in decreasing alertness. Second, we employ a hierarchical drift-diffusion model to tease apart and parameterise the different elements of the decision-making process and examine individually how alertness modulated them. Third, we use decoding at the temporo-spatial levels to understand how different elements of the decision-making processes (perceptual, evidence 
accumulation, motor implementation) may be implemented in the brain, and how such processes are modulated by fluctuations in alertness. Fourth, we connect the distinct behavioural parameters (evidence accumulation rate) of the decision making process to the neural processes, thereby capturing how the brain reconfigures, spatial and temporally, to maintain behavioural performance when challenged by decreased alertness.

\section{RESULTS}

\section{Behavioural evidence of decision making modulated by alertness}

We organized the results from direct and model-free to theoretically constrained. Error proportion, reaction times and subjective midline crosses are described as direct measures to evaluate the effects of alertness, while signal detection and drift-diffusion models theoretically constrain the interpretation of its parameters to perceptual, central and motor processes sequentially occurring during spatial decision making. We further organized the brain analyses similarly, using multivariate decoding to widely characterise the spatiotemporal neural signatures of the decision and, constrained by the behavioural results, we map the neural dynamics of evidence accumulation in full wakefulness and low alertness. This approach allowed for both, neurobehavioural characterization and hypotheses-driven evaluation, of the effects of low arousal on perceptual decision-making.

\section{Error-proportion modulated by alertness}

We first used multilevel modelling to understand how the errors made by each participant in an auditory tone-localization task (Figure 1A) was influenced by the stimulus presented ('left' or 'right' auditory tone) and the state of the participant ('alert' or 'drowsy'). We defined 4 different multilevel (linear mixed) models where errors were modulated by various combinations of stimulus and alertness states (see methods section). The analysis of variance table of the winning model shows that both alertness $(\mathrm{F}(1,95.07)=14.04, \mathrm{p}<0.0005)$ and stimulus type $(\mathrm{F}(1,95.07)=18.80, \mathrm{p}<0.00005)$ have an effect on error-proportion. Further, there was a reliable interaction between alertness levels and stimulus type $(\mathrm{F}(1,95.07)=6.88, \mathrm{p}<0.05)$. Next, post-hoc analysis revealed a reliable difference between alert and drowsy conditions for left stimuli $(\mathrm{p}<0.00005)$, and not for right stimuli $(\mathrm{p}=$ 0.859). These behavioural results (Figure 1B) replicate the findings of (25) in an independent study, task design, alertness measure (and lab), reporting an increase in location assignment errors on tones from the left side of the midline when people became drowsy. 
A
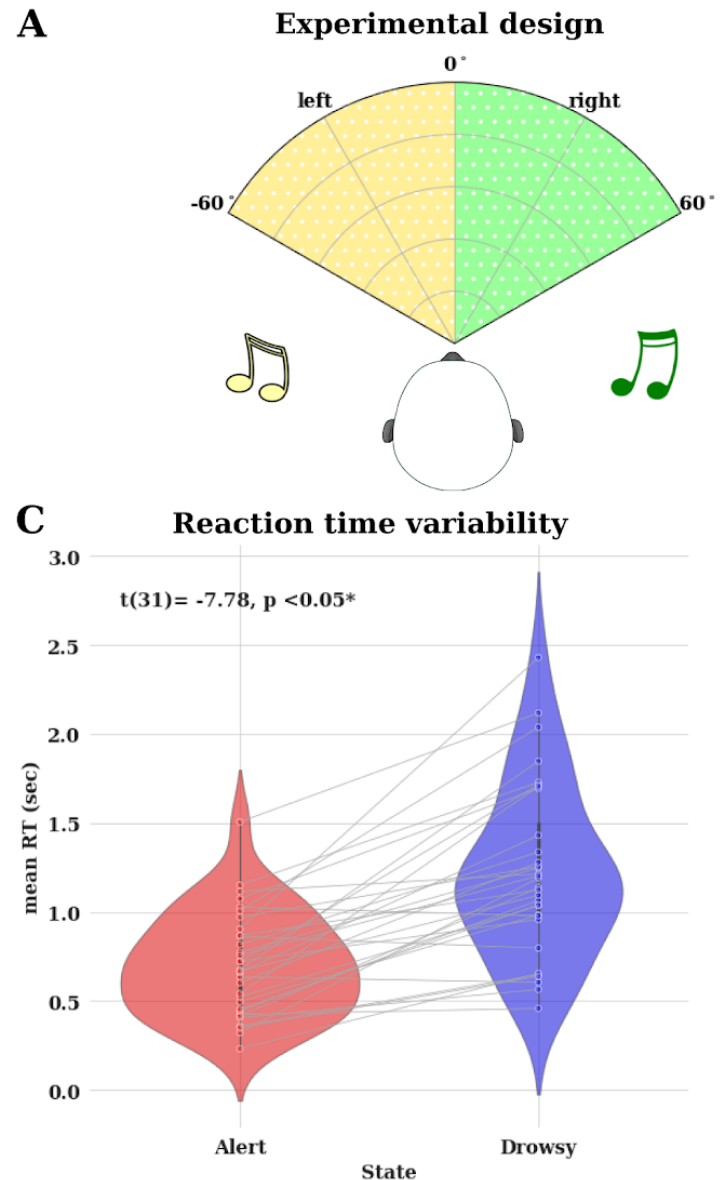

B

\section{Error-proportion}

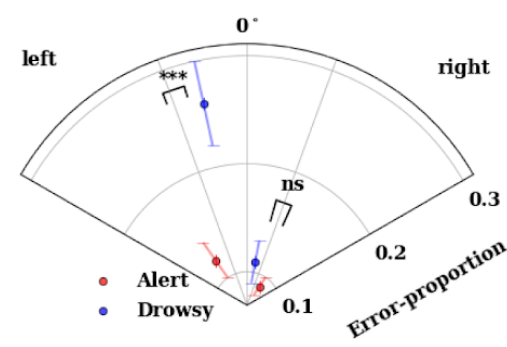

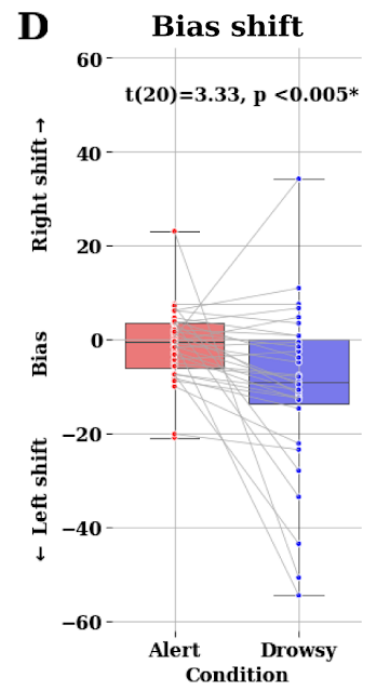
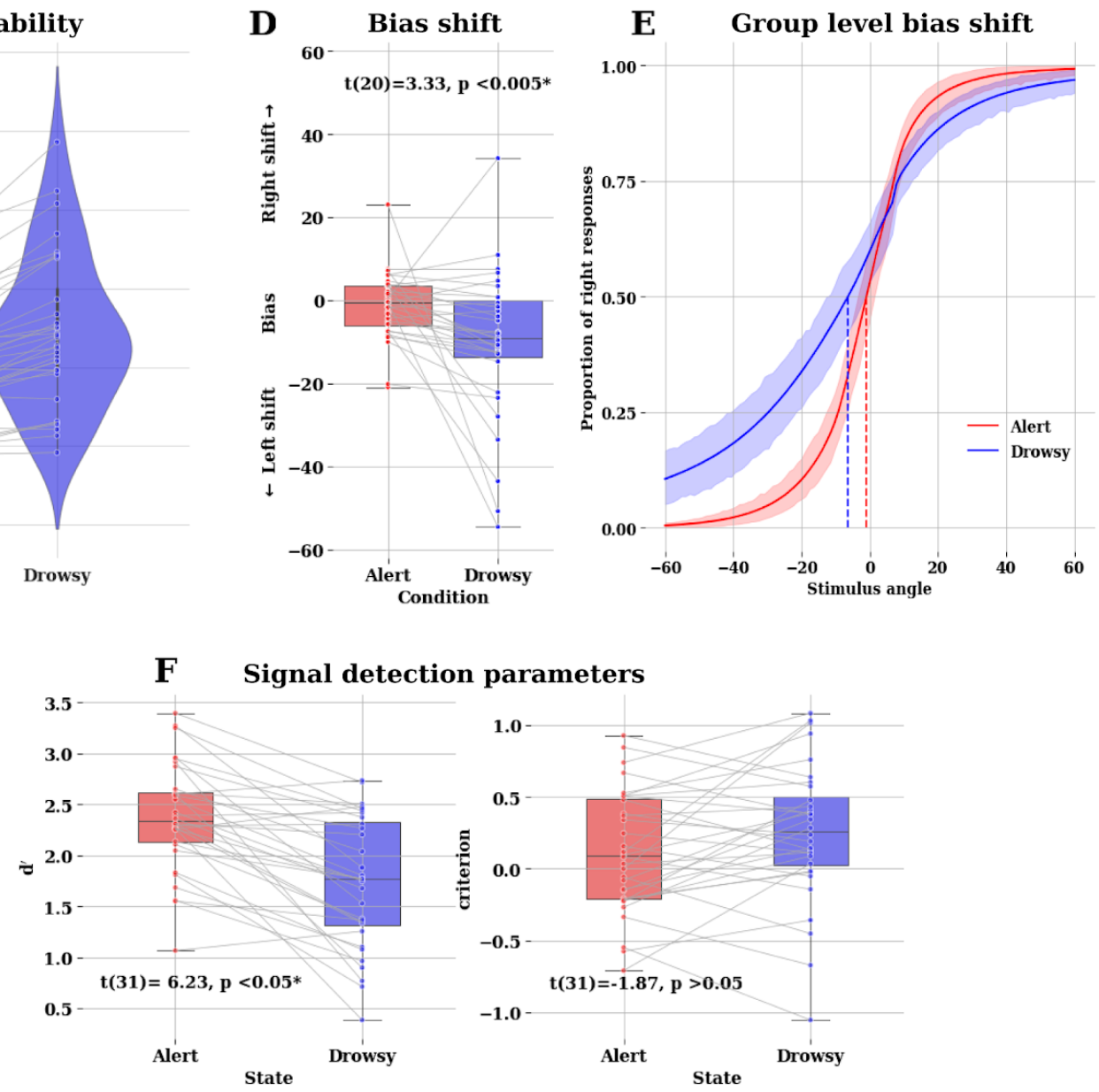

154 Figure 1: Auditory spatial attention task: A) participants had to localize the direction of auditory tones coming from left and right side of the midline. B) proportion of errors committed in alert and drowsy periods across left and right stimuli. Multilevel modelling reveals that error rates depend on stimulus type (left,right) and state of participant (alert,drowsy). Post-hoc tests indicate that error proportion is reliably modulated by alertness but only for left stimuli. ${ }^{* * *}$ indicates $\mathrm{p}<0.00005$, ns indicates not significant (or not reliable), error bars indicate standard error of the mean. C) mean reaction times for individual participants. Reaction times are variable and slower under drowsy conditions. D) spatial bias value per participant (using psychometric fits), bias level shifts towards the left side (indicating more left errors) for most participants. Negative bias 
162 values indicate shifts in the subjective midline towards the left and positive values indicate shift towards the right. E) group level psychometric fits indicate the shift in subjective midline (dotted lines), shaded regions are confidence interval bounds. F) signal detection analysis shows that only d' (sensitivity) seems modulated by alertness while criterion remains unaffected.

Reaction times are modulated by alertness

Second, we aimed to quantify the modulation in the response profiles (reaction times) of individual participants by alertness levels. A paired samples t-test with $\mathrm{t}(31)=-7.78, \mathrm{p}<0.05$ revealed a reliable effect of alertness on reaction times. These behavioural results (Figure 1C) converge with the original findings from Hori (23) indicating slower reaction times under lower levels of alertness, and

172 in agreement to all our previous work $(7,18,25-27)$.

\section{Subjective midline modulated by alertness}

Third, we used psychophysics to quantify the modulation of subjective midline per participant by alertness levels. For this purpose we fit a cumulative normal function (see methods section) to the proportion of rightward responses (per participant) under each stimulus condition from $-60^{\circ}$ to $60^{\circ}$ from the midline under both, alert and drowsy periods. The fit was weighted by the number of trials (responses) in each stimulus condition. The mean of the function referred to as 'bias' is the subjective midline or spatial bias (where participants have 0.5 chance of pressing left or right responses). Most participants (Figure 1D) had their bias point shifted to the left (as they became less alert: drowsy), indicating more left errors (as they overestimate the right side of space). A small proportion of participants had bias points shifted to the right. Overall, a paired samples t-test $t$ (20) $=3.33, \mathrm{p}<0.005$, revealed a reliable difference in bias points between alert and drowsy periods. Further, we also plotted (Figure 1E) the mean of the psychometric fits of individual participants to show that the overall subjective midline has shifted to the left at the group level.

\section{Signal detection parameters modulated by alertness}

Fourth, we used signal detection theory to understand the factors that modulate decision making under varying levels of alertness (Figure $1 \mathrm{~F}$ ). d'(sensitivity) was modulated by alertness with $\mathrm{t}(31)=$ 6.23, $\mathrm{p}<0.05$ however criterion (response bias) did not show reliable differences, $\mathrm{t}(31)=-1.87$, $\mathrm{p}>0.05$. This suggests that internal representations in the brain in terms of sensory/perceptual and noise distributions are modulated by alertness levels. Further, the lack of reliable differences in the response bias also suggests that participants were not arbitrarily pressing right responses for

To summarise, the behavioural results hint that the first two stages of perceptual, evidence accumulation process are affected by decreasing arousal and that the final stage of motor

200

\section{Decreased alertness modulates sequential sampling model parameters of 202 evidence accumulation of decision making}


204 Next, we aimed to quantify the different elements of the decision-making process using 205 drift-diffusion modelling. The drift-diffusion model captures the optimal procedure involved in 206 performing a 2-alternative forced choice (2AFC) task under sequential sampling framework. It 207 assumes that the observer accumulates evidence for one or other alternative in every other time 208 step (28), until integrated evidence reaches a threshold to decision (Figure 2A). The localization of 209 tones to the left and right side of space is essentially a 2-choice task with the participant always 210 forced to make a decision on the direction of tone. The model was implemented with a hierarchical 211 Bayesian procedure using hierarchical drift diffusion model (HDDM) (See methods section). For the 212 HDDMs, we fit the response of each participant instead of accuracy. This procedure is referred to as 213 Stimulus-coding, allows for the testing of several decision making parameters and is critical to 214 uncover response bias (20).

215

216 We examined 8 different variants of the model (see methods section), allowing the parameters 217 drift-rate (v) or bias-point (z) to vary based on state (alert or drowsy) or stimulus (left or right) 218 (Figure 2B,C). The winning model was chosen as the one with lowest deviance information criterion 219 (DIC) providing a balance between model fit and complexity. The winning model (\#8) was composed 220 of drift-rate (v) varied according to state (alert or drowsy) and stimulus (left or right), whilst bias-point (z) varied according to stimulus (left or right) only, furthering the claim that the changes in alertness place a higher burden in the evidence accumulation process (drift rate), and less on the motor implementation of the task. The winning model was then analysed for differences in posterior densities of parameters. For this purpose, we used a bayesian estimate which is fundamentally more informative and avoids the arbitrary choices like significance level and specific statistical tests used by the frequentist based methods (29).

We found in the winning model, the proportion of posterior overlap in the bias point (between left and right stimuli) was $24.2 \%$ (Figure $2 \mathrm{E}$ ). This indicates that the bias-point was not reliably different between the different stimuli. Further, the proportion of posterior overlap (between left and right stimuli) in the drift rate for alert trials was $13.8 \%$ this reduces to $3.0 \%$ for drowsy trials (Figure $2 \mathrm{H}$ ).

232 This indicates that the drift-rate (evidence accumulation rate) was reliably different between left 233 and right stimuli on drowsy trials (3.0\%) in comparison to the alert trials (13.8\%).

235 To summarise, the behaviour modeling results hint that the first two stages of perceptual encoding 236 and central evidence accumulation process (indicated by drift rate) are affected by decreasing 237 arousal. 
Decision making models based on evidence accumulation
A Drift-diffusion model
B shift in bias (z)
C shift in drift-rate (v)

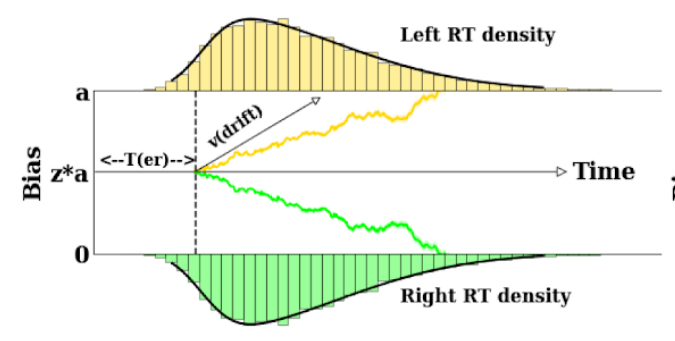

D
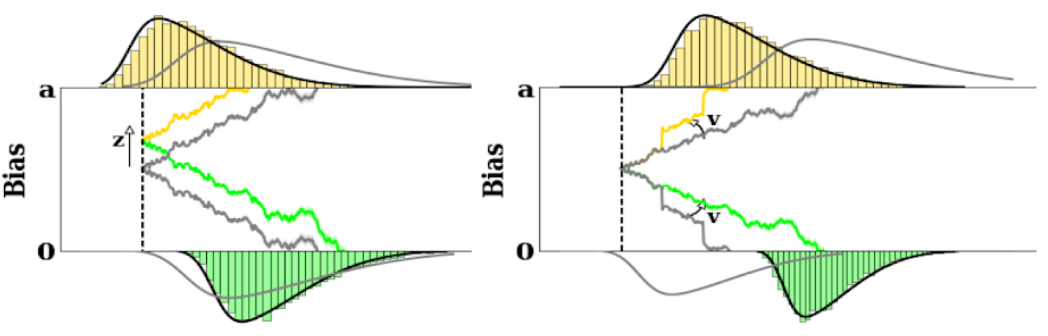

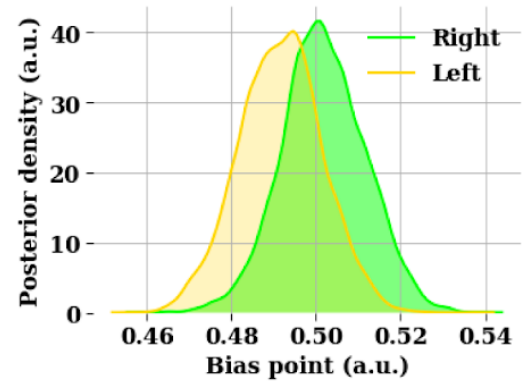

E bias point difference
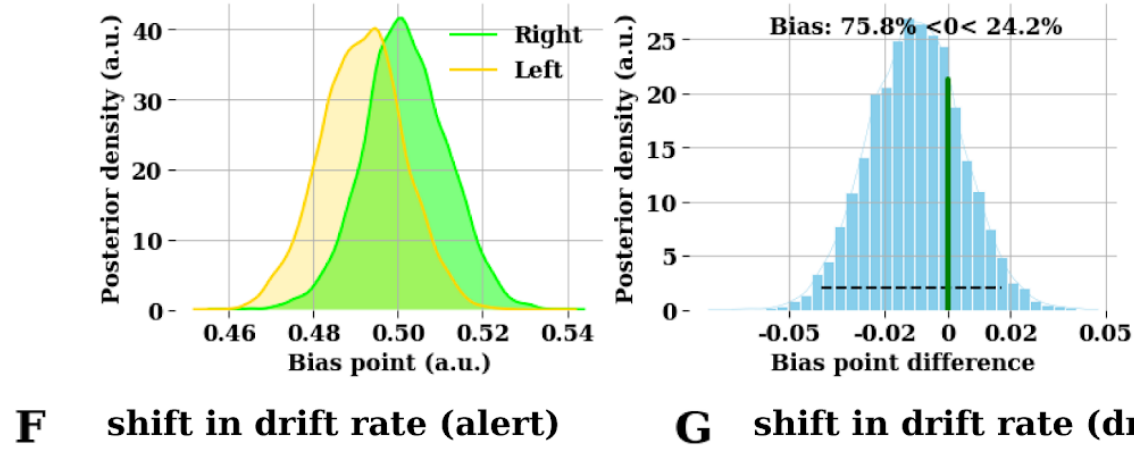

G shift in drift rate (drowsy)
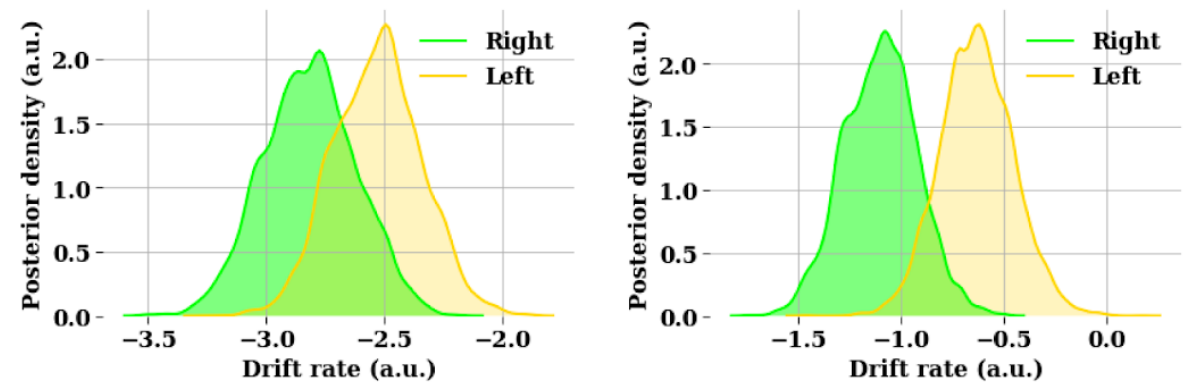

$\mathbf{H}$

drift rate difference

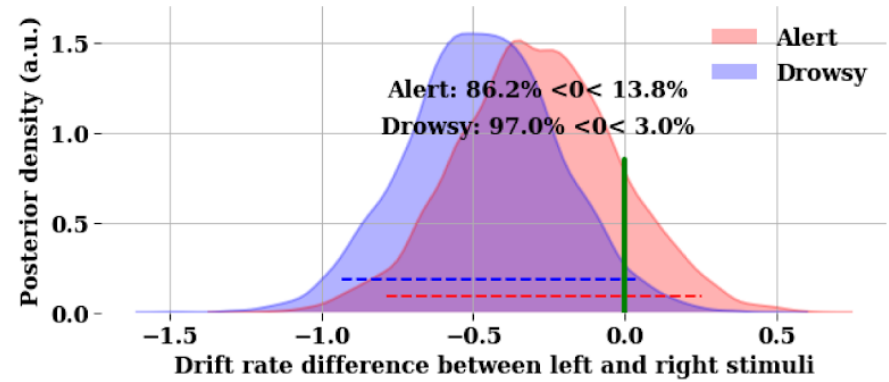

240 Figure 2: Evidence accumulation models: A) Drift-diffusion model accounts for the reaction time distributions of responses across left and right stimuli ('Stimulus Coding'). 'v(drift)' indicates evidence accumulation rate, 'a' indicates the boundary separation across left and right responses, ' $z$ ' indicates the bias point, usually $\mathrm{z}=0.5$ for unbiased responses. $\mathrm{B}, \mathrm{C}$ ) changes in response distributions explained by shift in ' $\mathrm{z}$ ', shift in ' $v$ ' respectively from alert to drowsy trials. grey lines indicate unbiased condition. D) posterior densities of ' $z$ ' for left and right stimuli in the winning model \#8. a.u. indicates arbitrary units. E) differences 
246 in the posterior densities indicate no evidence of bias point difference across stimuli. $F, G$ ) posterior densities of ' $v$ ' for left and right stimuli across alert and drowsy periods in the winning model \#8. $\mathrm{H}$ ) differences in the posterior densities of changes in drift rate indicate strong evidence in favour of change in drift rate across stimuli in the drowsy period compared to alert periods.

250

\section{Lower alertness delays the processing of spatial decision making}

253 Next, we aimed to uncover how the neural patterns in the EEG data are modulated by alertness 254 levels. Conventional event related potentials (ERP) analysis for this purpose, relies on a-priori 255 identified spatial locations or temporal segments to measure the differences across conditions. For data driven characterisation of the neural echoes of our experiment we decided to use time resolved decoding techniques that do not rely on such a-priori definitions and, further these techniques due to its multivariate nature, perform much better in detecting differences across experimental conditions (30).

\section{Spatial and temporal signatures involved in spatial localization across alert and drowsy periods}

263

264 First, we were interested in identifying the neural signatures involved in the performance of this task during alert condition. For this purpose, decoding involved in identifying the stimulus (X - left or right tone) presented from the EEG data $(\mathrm{Y})$. This process involves the identification of the $\mathrm{W}$ (classifier weights) that can produce the transformation, $\mathrm{Y}_{\mathrm{t}}=\mathrm{W}_{\mathrm{t}} \mathrm{X}_{\mathrm{t}}$ where ' $\mathrm{t}$ ' represents time (Figure $3 \mathrm{~A}, \mathrm{~B})$. The performance of the classifier (W) is evaluated by training and testing the data at each time-point ( $\mathrm{t}$ ) using area under the curve (AUC) as measure. In Figure 3C, the shaded region represents those periods reliably decoded $(\mathrm{p}<0.05)$ (see methods for more details).

We found that when participants were alert the decoding of stimuli (Figure 3D) started at $160 \mathrm{~ms}$ after the stimulus and reliably lasted until $730 \mathrm{~ms}$ (cluster permutation, p<0.05) with mean AUC of 0.57. The peak discriminatory power was at $280 \mathrm{~ms}$ (AUC =0.61). The average AUC between 200-300

$275 \mathrm{~ms}$ was $0.58 \pm 0.007, \mathrm{p}<0.05$, between $300-400 \mathrm{~ms}$ was $0.56 \pm 0.002, \mathrm{p}<0.05$. However when the participants became 'drowsy' the decoding of stimuli (Figure 3E) shifted to $420 \mathrm{~ms}$ after the stimulus was presented and lasted until $730 \mathrm{~ms}$ (reliable with cluster permutation, $\mathrm{p}<0.05$ ) with mean AUC of 0.54 . The peak discriminatory power was at $590 \mathrm{~ms}(\mathrm{AUC}=0.56)$. The average AUC between $420-500 \mathrm{~ms}$ was $\mathrm{M}=0.53 \pm 0.002$, $\mathrm{p}<0.05$, between $510-590 \mathrm{~ms}$ was $\mathrm{M}=0.54 \pm 0.004$, $\mathrm{p}<0.05$. This suggest that the processes related to the discrimination between left and right stimuli under drowsy conditions may cease to be directly informative to the decision in the early processes of sensory encoding and would only start later, around $\sim 400 \mathrm{~ms}$, to show neural differentiation.

283 Further to this, the lower discriminatory power points to a potentially less efficient (lower 284 decodability, longer time duration) process of central evidence accumulation, or to a different 285 neural implementation of the processes during low alertness. 
bioRxiv preprint doi: https://doi.org/10.1101/2020.07.23.218727; this version posted January 23, 2021. The copyright holder for this preprint (which was not certified by peer review) is the author/funder, who has granted bioRxiv a license to display the preprint in perpetuity. It is made available under aCC-BY-NC-ND 4.0 International license.

A

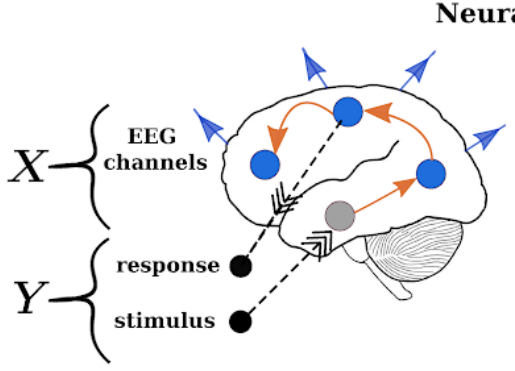

B

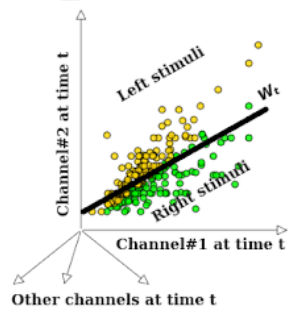

$\mathbf{C}$

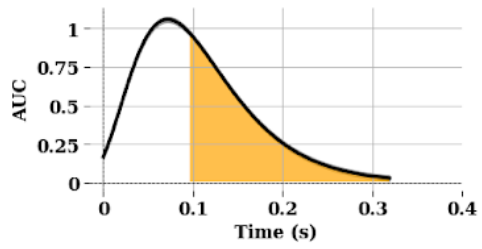

D

Decoding left vs right stimuli in alert periods

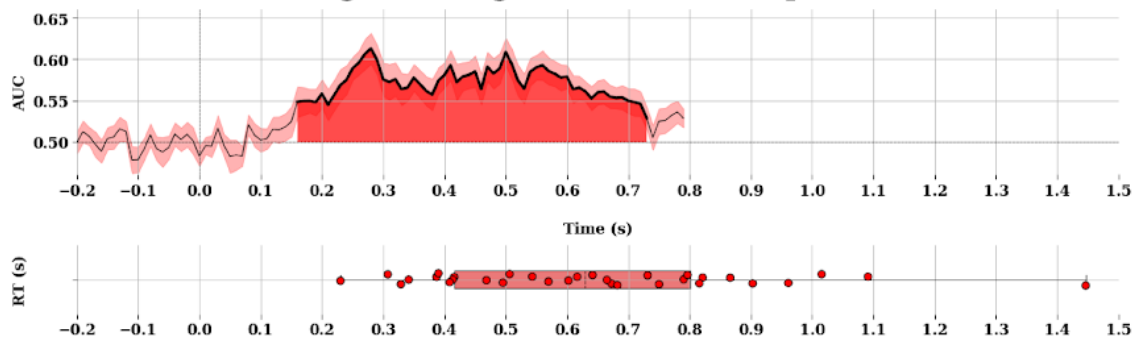

$\mathbf{E}$

Decoding left vs right stimuli in drowsy periods
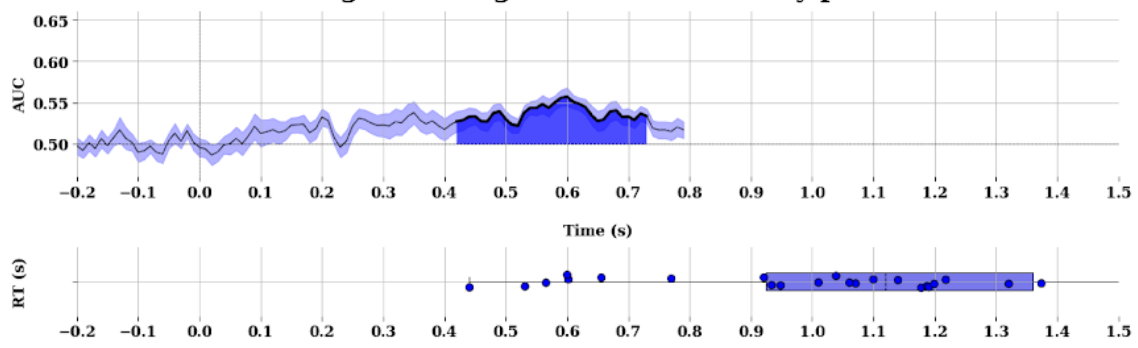

$\mathbf{F}$

Classifier patterns comparison

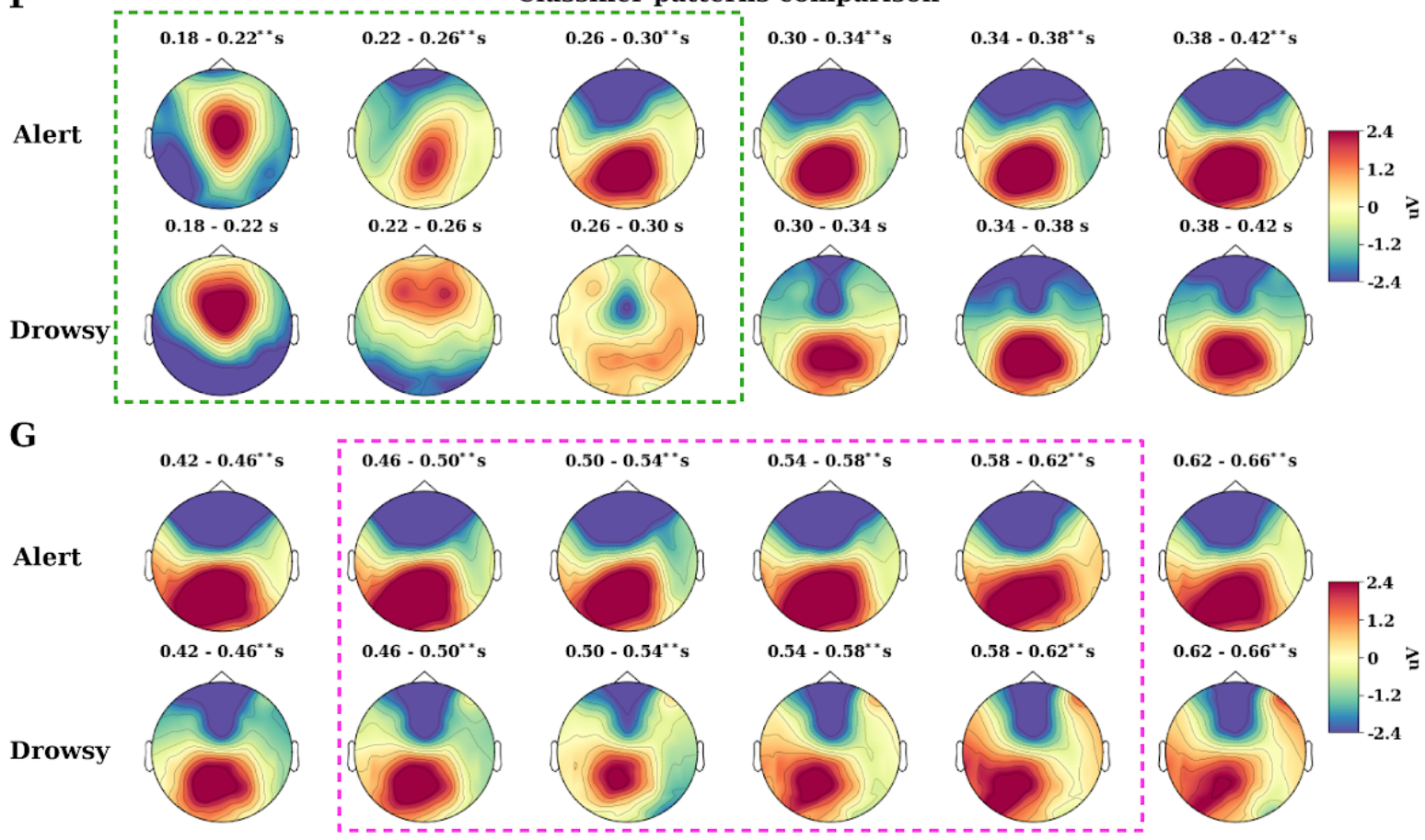


287 Figure 3: Temporal decoding (stimuli): A) decoding consists of identifying Y (responses or stimuli) from X (EEG data). The model thus consists of $\mathrm{Y}_{\mathrm{t}}=\mathrm{W}_{\mathrm{t}} \mathrm{X}_{\mathrm{t}}$, where ' $\mathrm{t}$ ' represents time and $\mathrm{W}_{\mathrm{t}}$ represents transformation (classifier weights). B) classifier weights $\mathrm{W}_{\mathrm{t}}$ are determined by the optimal separation of different classes (here left, right stimuli). C) decoding performance is assessed using the area under curve (AUC) where shaded regions represent reliably different time periods. D,E) AUC under alert and drowsy periods, where the classifier was trained to discriminate between targets of left and right stimuli. Shaded regions show time periods with reliable $(\mathrm{p}<0.05)$ discriminatory power. The mean reaction times $(\mathrm{RT})$ of participants are plotted below. F) comparison of coefficients of classifier patterns in the early time periods. The boxed region highlights topographical differences in the frontal, posterior parietal and central electrodes. G) comparison of coefficients of classifier patterns in the later time periods. The boxed region highlights topographical differences in the frontal and central electrodes. $* *$ indicates reliable discriminatory in the corresponding

Next, we plotted the coefficients of the classifier patterns (derived from classifier weights W) for further neurophysiological interpretation (31). We decided to compare the classifier patterns across early $(<300 \mathrm{~ms})$, and later $(>400 \mathrm{~ms})$ time periods. To compare patterns in the early time periods across 'alert' and 'drowsy' periods, we plotted the same for every $40 \mathrm{~ms}$ between $180 \mathrm{~ms}$ to $420 \mathrm{~ms}$ in Figure 3F. The green box indicates clear differences across alert and drowsy in the early time periods. For the alert periods, the pattern between 180-220 ms indicates a strong involvement of signal in the fronto-central electrodes, whereas in the corresponding time periods under drowsy, the pattern seems to have shifted to more frontal electrodes although its contribution may be minimal as it was not reliably decodable. Under alert periods, the pattern shifts to more posterior regions (centro-parietal electrodes in the right side of the scalp) between $220 \mathrm{~ms}$ to $260 \mathrm{~ms}$ whereas under drowsy the pattern stays in the frontal electrodes itself, still not reliably decodable in the unconstrained voltage decoding. Further from 260-300 ms the pattern shifts to more parietal and occipital sites under alert periods and is only weakly parietal in the drowsy periods. The comparison

314 of the classifier patterns across alert and drowsy periods (in early time periods), reveals different

315 topographies that point to a clear differential processing of information that would further map onto shifted perceptual and central evidence accumulation stages of cognitive processing (2) possible affecting both, perceptual encoding and central accumulation. To compare patterns in the later time periods across 'alert' and 'drowsy' periods, we plotted the same for every 40 ms between $420 \mathrm{~ms}$ to $660 \mathrm{~ms}$ in Figure 3G. The magenta box indicates clear differences across alert and drowsy in the later time periods. Particularly, the patterns in the alert periods have a lower frontal activity and higher posterior activity, whereas in the drowsy periods, the activity in both the frontal and posterior sites is much more localised although decodability is overall lower. Although only suggestive, this change in the decodability intensity and distribution suggest a delayed processing 


\section{Spatio temporal clustering of classifier patterns in alert and drowsy periods}

$\mathbf{A}$

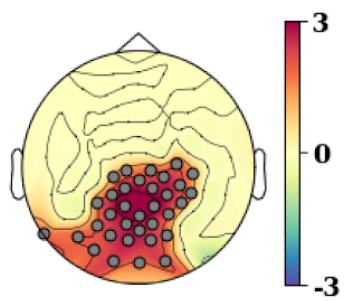

F-stat (mean)

B

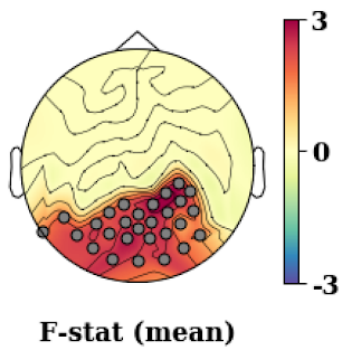

328

C

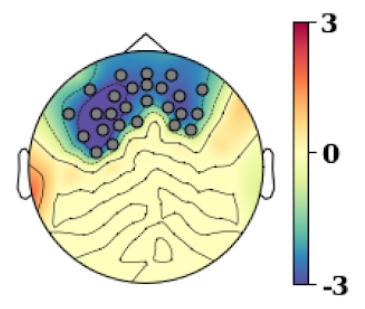

F-stat (mean)

D

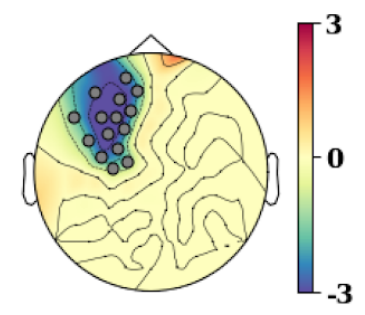

F-stat (mean)
Alert > Drowsy : Cluster\# 1 (0.16 - 0.32 s)

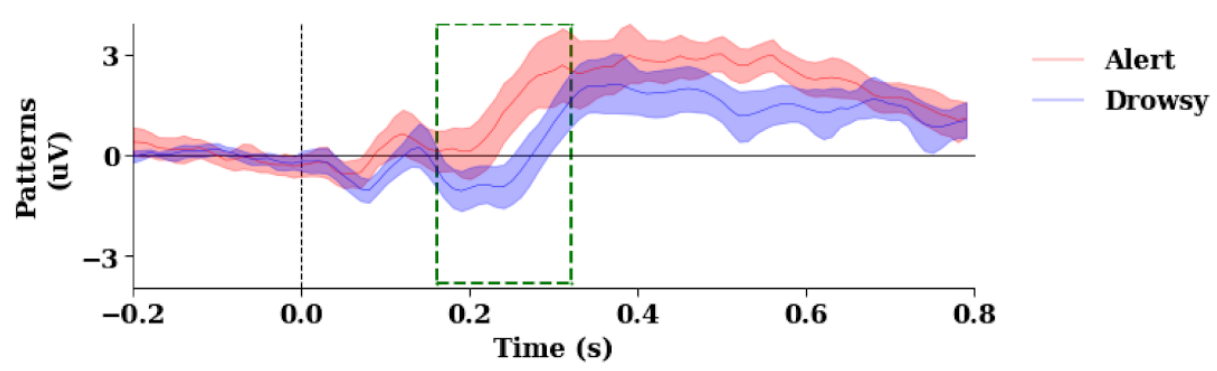

Alert > Drowsy : Cluster\#2 (0.44 - 0.62 s)

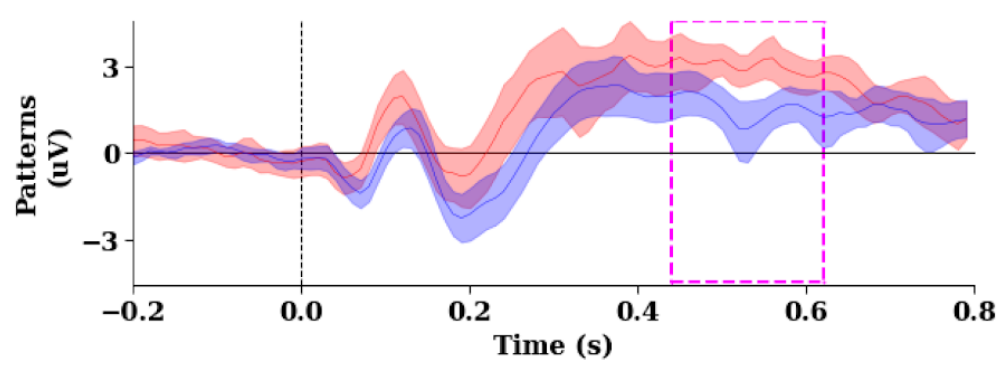

Drowsy > Alert : Cluster\#3 (0.18 - 0.31 s)

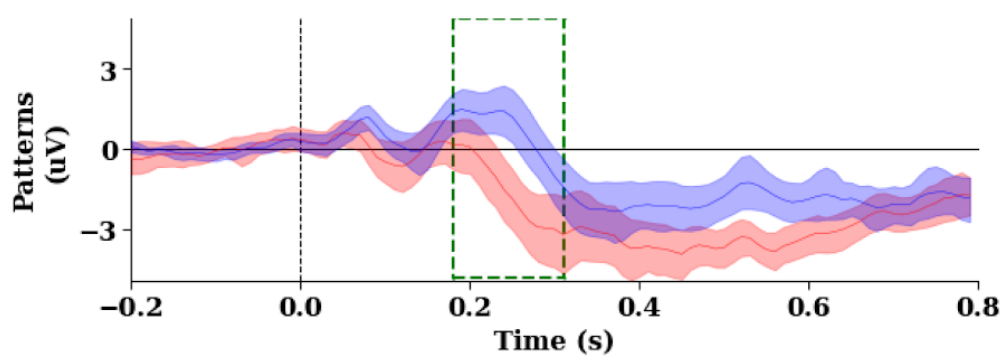

Drowsy > Alert : Cluster\#4 (0.39 - 0.62 s)

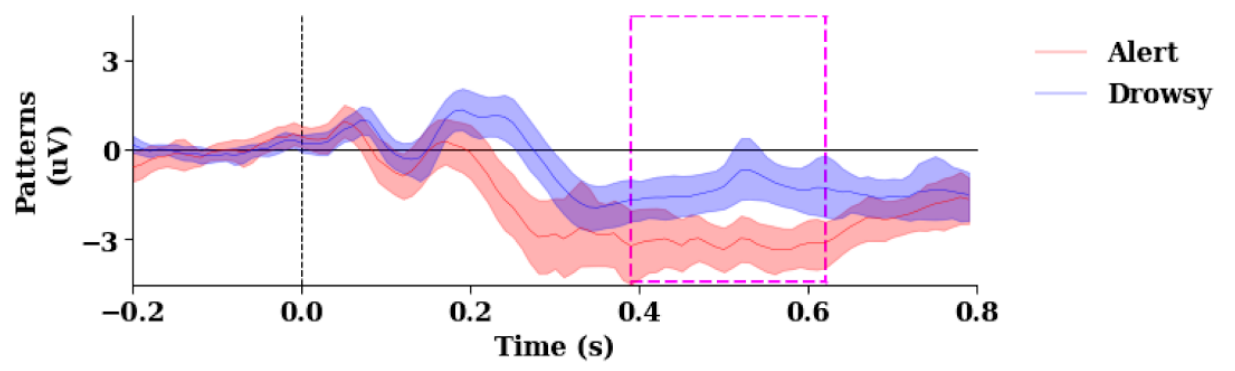

Figure 4: Classifier pattern differences: To identify differences between the classifier patterns in Figure 3 $(\mathrm{F}, \mathrm{G})$ we performed spatio-temporal clustering. A) Cluster\#1 indicates early periods (160 - $320 \mathrm{~ms}$ after stimulus) where alert activity is higher than drowsy periods. B) Cluster\#2 indicates later periods (440 - $620 \mathrm{~ms}$ after stimulus) where alert activity is higher than drowsy periods. In terms of spatial locations, the early and later clusters differ in right parietal sites, central, middle electrode sites. C) Cluster\#3 indicates early periods (180 - 310 ms after stimulus) where drowsy activity is higher than alert periods. D) Cluster\#4 indicates later 
periods (390 - $620 \mathrm{~ms}$ after stimulus) where drowsy activity is higher than alert periods. In terms of spatial locations, the early and later clusters differ in left frontal sites, middle electrode sites.

The descriptive analysis of the classifier patterns indicate differences between alert and drowsy periods. To establish the spatial and temporal signatures of such differences we performed a cluster permutation test and identified regions where activity patterns in alert periods are different from drowsy periods. This analysis resulted in the identification for four clusters.

a) Cluster \#1 (alert activity > drowsy activity): indicates early periods (160 ms to $320 \mathrm{~ms}$ ), wherein activity is concentrated in parietal, central, posterior electrode sites. These spatial patterns are likely to be involved in early perceptual/evidence accumulation processes during the alert periods, as the majority of responses only start occurring from $400 \mathrm{~ms}$ onwards.

b) Cluster \#2 (alert activity > drowsy activity): indicates later periods (440 ms to $620 \mathrm{~ms}$ ), wherein activity is concentrated in occipital, parietal, central, posterior electrode sites. These spatial patterns are difficult to interpret as the reaction times overlap with the corresponding time periods. Hence this decoding pattern could be an amalgamation between central evidence accumulation and motor implementation processes.

c) Cluster \#3 (drowsy activity > alert activity): indicates early periods (180 ms to $310 \mathrm{~ms}$ ), wherein activity is concentrated in frontal electrode sites. These spatial patterns are likely to be involved in early perceptual/sensory encoding processes during the drowsy periods, as the majority of responses only start occurring from $900 \mathrm{~ms}$ onwards. The temporal neurodynamics show delayed patterns in drowsy compared to awake (3 DE and $4 \mathrm{AC}$ ).

d) Cluster \#4 (drowsy activity > alert activity): indicates later periods (390 ms to $620 \mathrm{~ms}$ ), wherein activity is concentrated in left frontal electrode sites. These spatial patterns are likely to be involved in central evidence accumulation related processes. However it is difficult to interpret as the corresponding time periods during alert overlap with response related activity.

To summarise, these results indicate differences in spatial patterns related to early sensory/evidence accumulation related process (cluster \#1,3) across alert and drowsy periods. The next step is to identify and tease apart motor preparation related processes occurring during later time periods.

\section{Spatial and temporal signatures of the motor implementation of the decision across alert and drowsy periods}

In classic cognitive processing model frameworks (Figure s1), motor implementation occurs after the central evidence processes, when information of the decision is routed to the premotor network. We preprocessed the EEG data now locked to responses (see methods section) and decoded the 
376 response hand itself (irrespective of the stimulus presented). These response related patterns thus

377 would depict information related to the motor implementation process irrespective of the decision

378 related processes.

\section{A}

\section{Decoding response hand locked to responses}

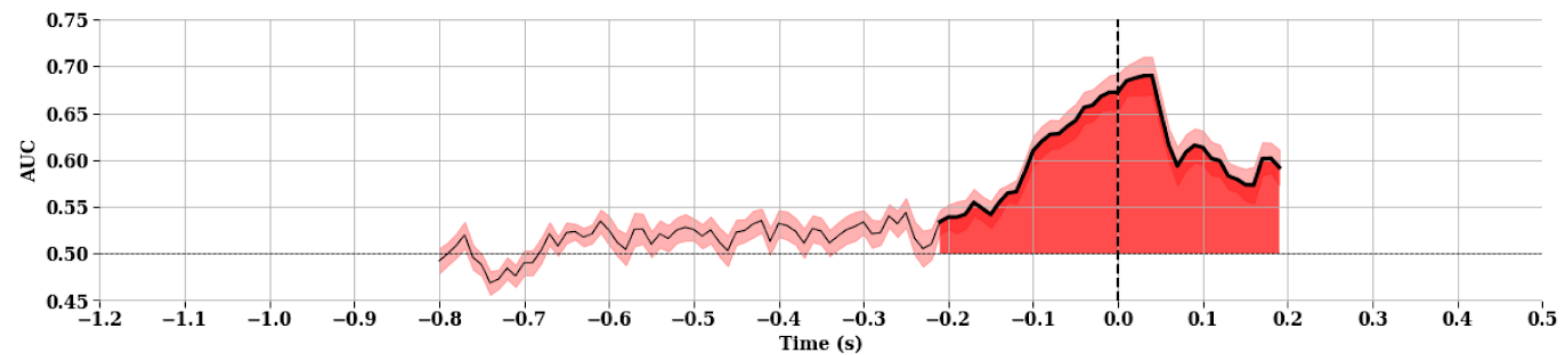

B

Decoding left vs right responses in drowsy periods

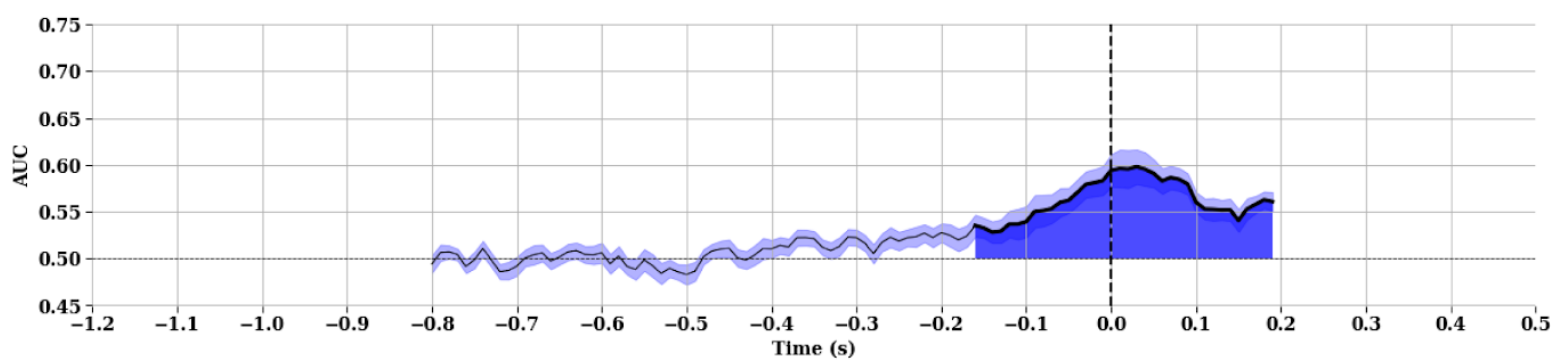

Spatio temporal clustering of response patterns across alert and drowsy periods

C

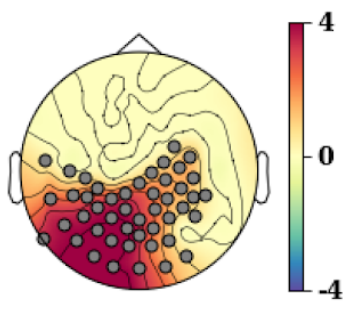

F-stat (mean)

$\mathbf{D}$

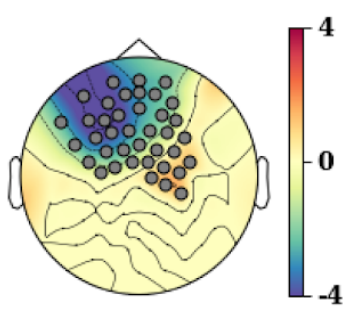

F-stat (mean)
Alert > Drowsy : Cluster\# 1 (-0.19 to 0.19 s)

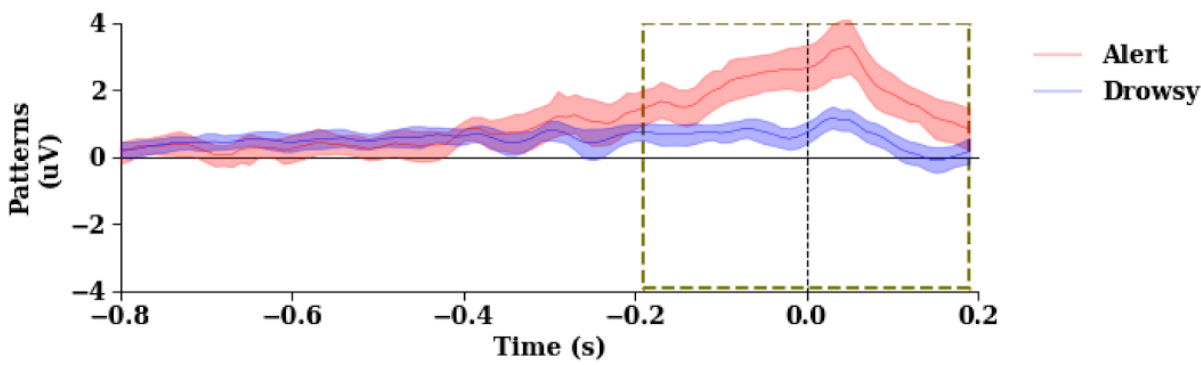

Alert > Drowsy : Cluster\#2 (-0.29 to 0.19 s)

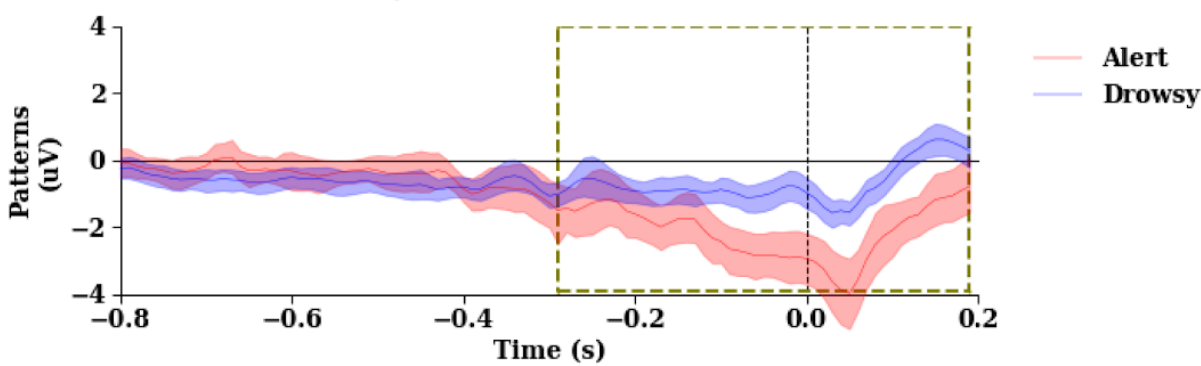

Figure 5: Temporal decoding (responses): To identify differences in motor preparation (response related), we decoded the left and right button press events irrespective of stimuli presented. A,B) AUC under alert and drowsy periods, where the classifier was trained to discriminate between targets of left and right button 
presses. Shaded regions show time periods with reliable $(\mathrm{p}<0.05)$ discriminatory power. The time point of 0 corresponds to the actual button press event. The ramp up of the AUC under both conditions starting from $210 \mathrm{~ms}$ (alert), 160ms (drowsy) before the actual response indicates the ability of the decoder to detect neural signatures related to responses. Spatio-temporal clustering of differences in classifier patterns produces two clusters with similar time periods in contrast to the clusters (with early and later time periods) produced by stimuli related classifier patterns. C) Cluster\#1 indicates periods (-190 to $190 \mathrm{~ms}$ ) where alert activity is higher than drowsy periods. D) Cluster\#2 indicates periods (-290 to $190 \mathrm{~ms}$ ) where drowsy activity is higher than alert periods. In terms of spatial locations, the clusters differ in frontal, central, middle, parietal electrode sites. The presence of a single cluster with similar time periods across alert and drowsy periods indicate that motor related processes start appearing at similar time periods and hence do not change with modulations in alertness levels.

The decoding performance: AUC (Figure 5A,B) starts ramping up from -210ms (alert), -160ms (drowsy) from the response time, and increases when the button is actually pressed at 0 sec, reaching the peak soon after. In both cases, awake and drowsy, the neural decoding results lasts till 190ms. These results are in line with motor implementation related processes commonly reported around $200 \mathrm{~ms}$ before the actual motor response, gradually ramping up to produce the response itself. It is also interesting to note that the AUC for alert is always higher than drowsy, indicating less variability in neural concerted activity, leading to better classifier performance without time span differences.

The classifier patterns related to responses are presented in Figure s5. To establish the spatial and temporal signatures of differences in motor related processes we performed a cluster permutation test and identified regions where activity patterns in alert periods are different from drowsy periods. This analysis resulted in the identification of two clusters.

a) Cluster \#1 (alert activity > drowsy activity): indicates periods (-190 ms to $190 \mathrm{~ms}$ ), wherein activity is concentrated in premotor, parietal, posterior electrode sites.

b) Cluster \#2 (drowsy activity > alert activity): indicates periods (-290 ms to $190 \mathrm{~ms}$ ), wherein activity is concentrated in premotor, frontal electrode sites.

The existence of a single cluster (in time) across both alert and drowsy periods indicate that motor related processes do not vary in time with modulations in alertness levels. Furthermore for both alert and drowsy periods the start of the decoding happens during similar time periods around -200ms and last till similar time periods $\sim 190 \mathrm{~ms}$.

To summarise, these results indicate that motor preparation related processes start and end at similar time periods during both alert and drowsy trials, suggesting the differences in motor preparation process may not be critical to the changes in decision making in low arousal when compared to early sensory and evidence accumulation related processes.

The above analysis related to decoding of stimuli and responses indicate that sensory encoding, central evidence accumulation related processes are modulated by alertness. However the decoding 
444 It can be expressed as

457 Next, we plot the regression patterns in alert and drowsy periods. The patterns in the alert periods 458 (Figure 6B) indicate that the topography focuses initially in the fronto-central electrodes (150 ms to $459250 \mathrm{~ms}$ ), which then shifts to centro-parietal electrodes (250 ms to $350 \mathrm{~ms}$ ), similarly to the 460 classifier analysis (decoding patterns from figures $3 \mathrm{~F}$ and $3 \mathrm{G}$ ). The patterns in the drowsy periods 461 (Figure 6C) indicate that the topography initially focuses on the frontal electrodes (though weaker 462 compared to alert periods) in the interval from $150 \mathrm{~ms}$ to $250 \mathrm{~ms}$. Further the patterns shift to more 463 central electrodes (again weaker when compared to alert periods) in the interval from $250 \mathrm{~ms}$ to 350 $464 \mathrm{~ms}$.

analysis in the later parts could be confounded by response related processes occurring during alert periods. Hence we devised the next step of the analysis wherein we use parameters derived from the drift diffusion modelling, to understand how different elements of the sensory encoding and evidence accumulation are represented in the neural domain. This analysis is determined by the drift diffusion model, based in a mathematical implementation of decision making theory.

\section{Neural Signatures of evidence accumulation of decision making modulated by alertness}

To take advantage of the information gained by the drift diffusion modelling we decided to capture the neural implementation of the evidence accumulation parameters in the decision making process, and how it might differ in wakefulness and low alertness periods. We specifically aimed at capturing the change in the neurobehavioural dynamics of the evidence accumulation process in the awake and drowsy periods. To establish this we developed a novel method based on the drift-diffusion analysis shown earlier. First, we used the winning model (\#8) and computed trial-by-trial drift parameter $(d v)$. Second, we used $d v$ as a dependent variable and regressed the same against ERP data (see methods section).

The above equation can be written in patsy form as below.

$$
\left.d v \sim \text { ERP : C(state, Treatment }\left({ }^{\prime} \text { Alert }{ }^{\natural}\right)\right): C\left(\text { stim, Treatment }\left({ }^{(} \text {Right }{ }^{\natural}\right)\right)
$$

Here, $d v$ represents trial-by-trial drift-rate, ERP represents z-scored ERP data per trial per time point per electrode, state represents alertness levels ('alert' or 'drowsy'), stim represents stimulus types ('left' or 'right'). Further we compute the proportion of the overlap of the posterior distributions of the trace obtained for both left and right stimuli separately under alert and drowsy condition (Figure 6A). This analysis is repeated for each participant and we obtain regression patterns (similar to the classifier patterns) per participant. 


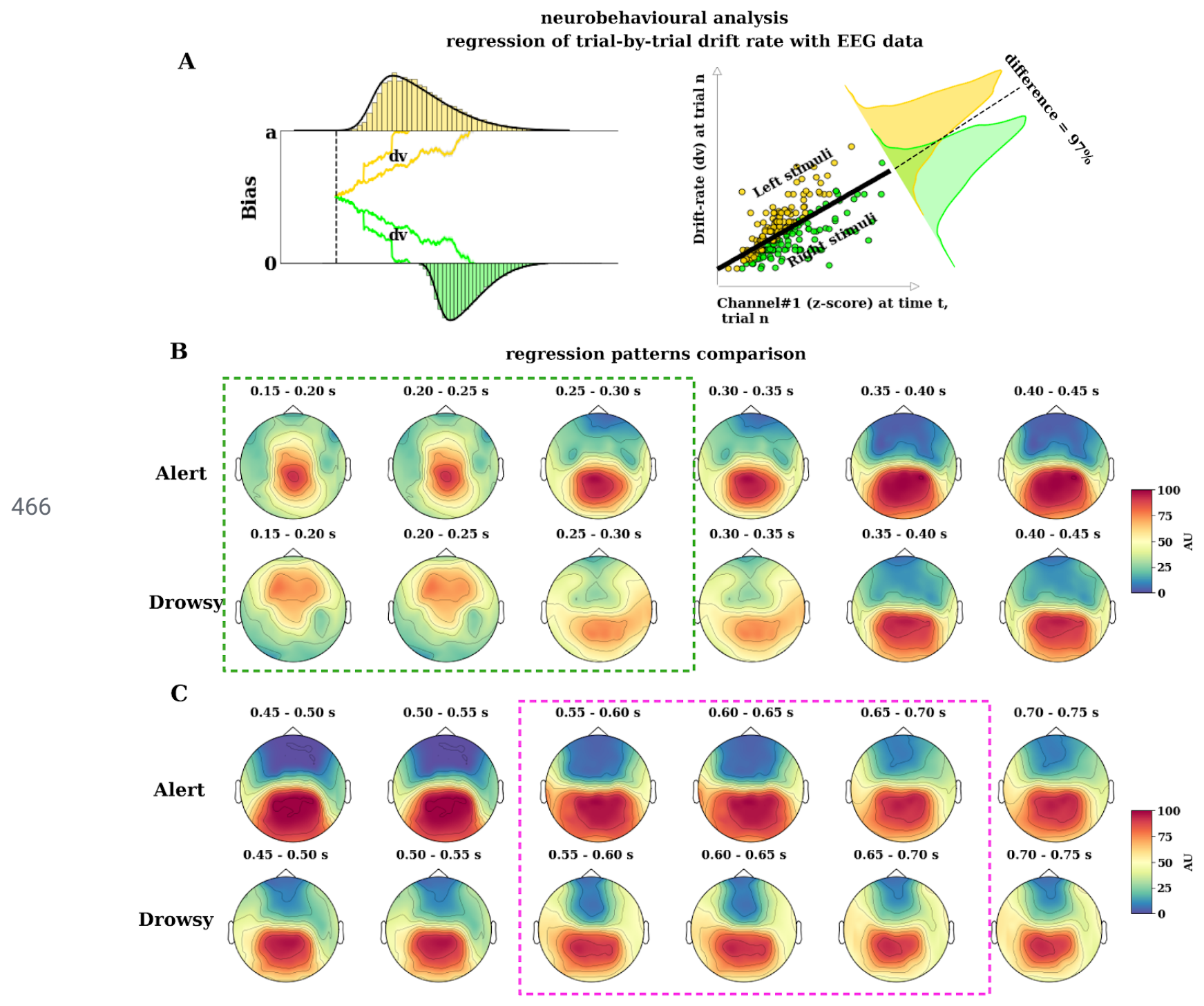

468 Figure 6: Regression patterns: A) trial by trial drift rate (dv) was regressed against EEG data (z-scored and averaged per $50 \mathrm{~ms}$ ), the difference in the posterior of regression rate was quantified per electrode per time-point per participant. B,C) mean regression patterns in alert and drowsy periods projected in electrode space show a classic centro frontal to parietal progression from $150 \mathrm{~ms}$ to $500 \mathrm{~ms}$. The green box highlights topographical differences in the frontal, middle, central parietal electrodes (in early time periods) and magenta box highlights the frontal, parietal electrode sites (in later time periods). 


\section{Spatio temporal clustering of regression patterns in alert and drowsy periods}

A

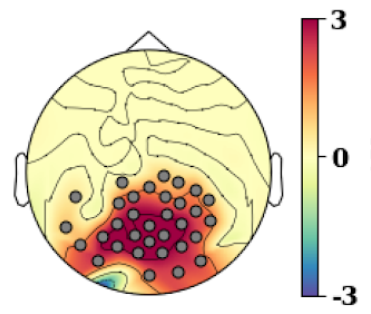

F-stat (mean)

B

475

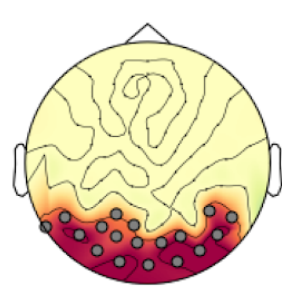

F-stat (mean)

C

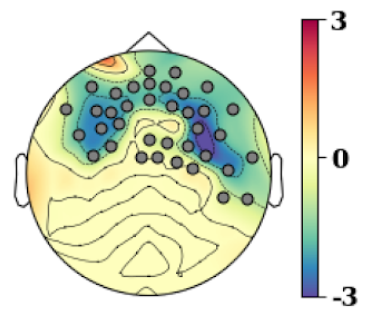

F-stat (mean)

D

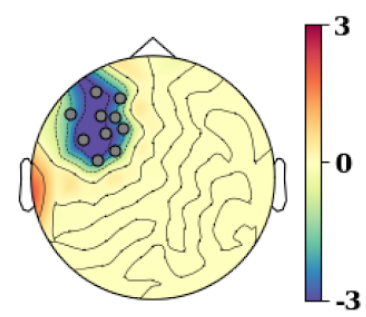

F-stat (mean)

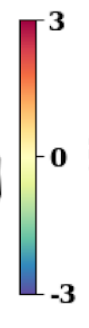

Alert > Drowsy : Cluster\# 1 (0.07 - 0.28 s)

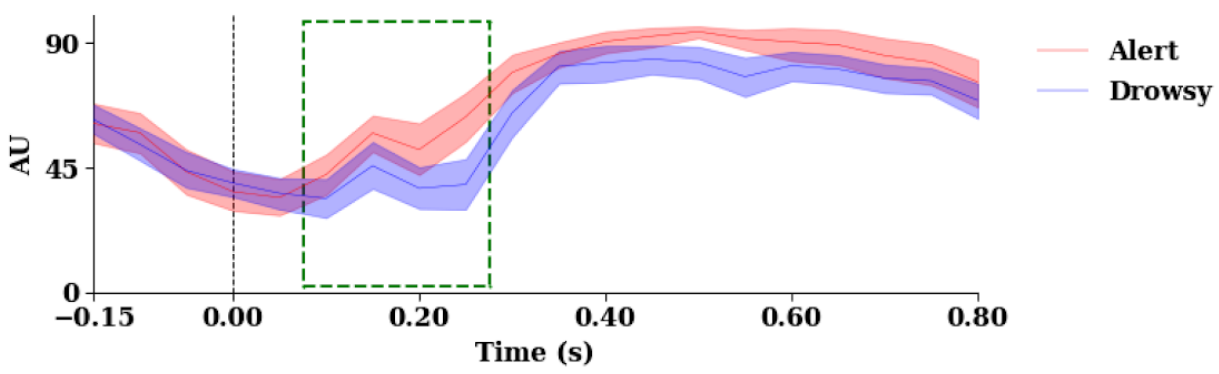

Alert > Drowsy : Cluster\#2 (0.47 - 0.72 s)

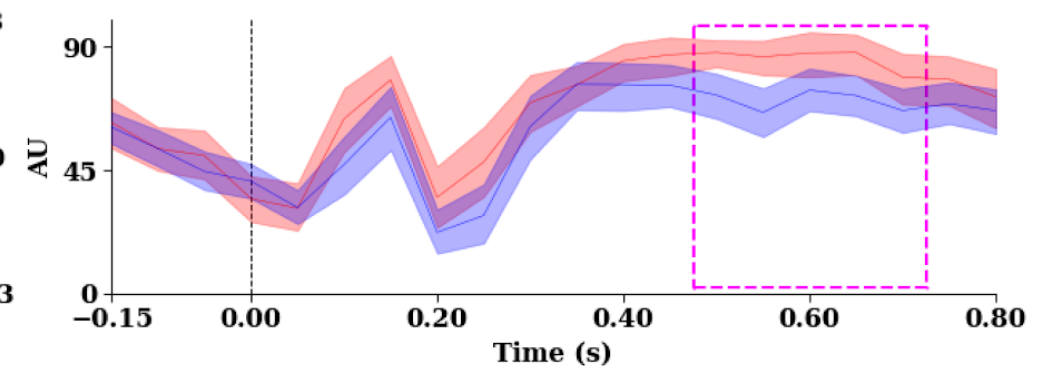

Drowsy > Alert : Cluster\#3 (0.07 - 0.33 s)

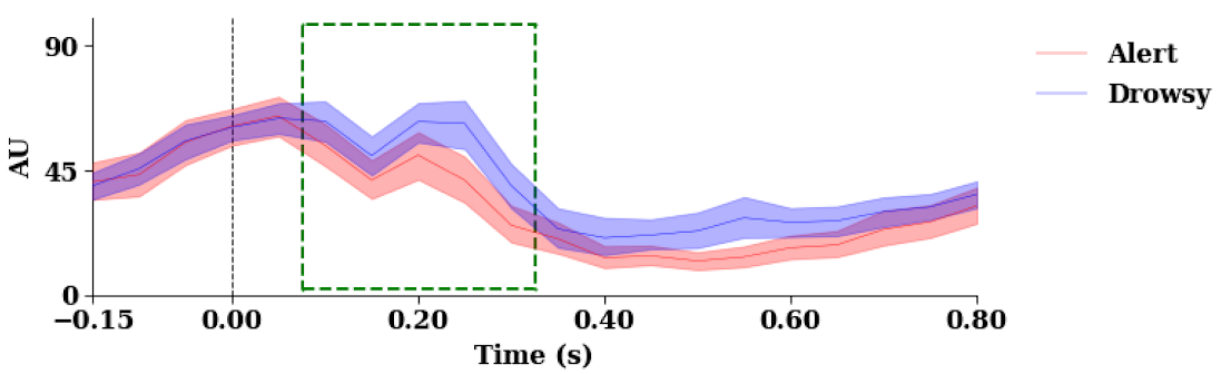

Drowsy > Alert : Cluster\#4 (0.38 - 0.68 s)

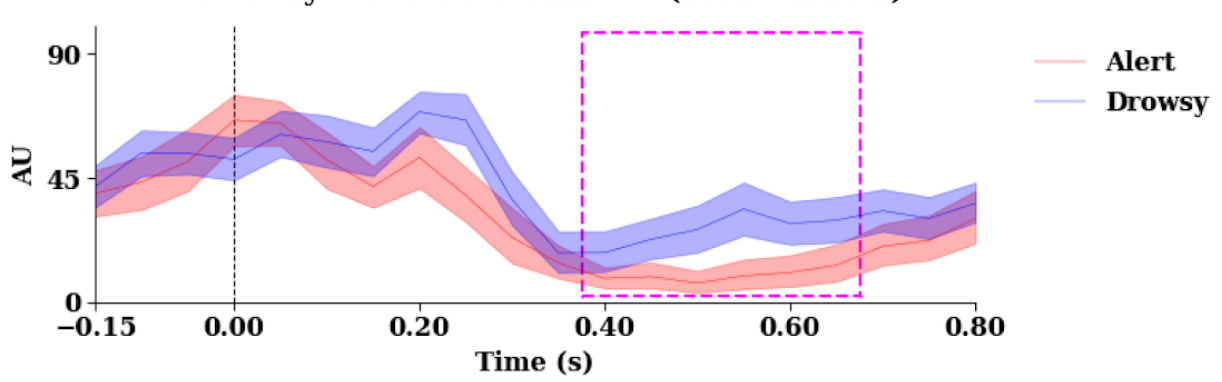

477 Figure 7: Regression pattern differences: spatio-temporal clustering of differences in regression patterns shown in Figure 6 (B,C) between alert and drowsy periods. A) Cluster\#1 indicates early periods (70 - $280 \mathrm{~ms}$ after stimulus) where alert activity is higher than drowsy periods. B) Cluster\#2 indicates later periods (470 $720 \mathrm{~ms}$ after stimulus) where alert activity is higher than drowsy periods. In terms of spatial locations, the early and later clusters differ in right parietal sites, central, middle electrode sites. C) Cluster\#3 indicates early periods (70 - $330 \mathrm{~ms}$ after stimulus) where drowsy activity is higher than alert periods. D) Cluster\#4 indicates 
483 later periods (380 - 680 ms after stimulus) where drowsy activity is higher than alert periods. In terms of spatial locations, the early and later clusters differ in left frontal sites, middle electrode sites.

485

The descriptive analysis of the regression patterns indicate differences between alert and drowsy periods. To establish the spatial and temporal signatures of such differences we performed a cluster permutation test and identified regions where activity patterns in alert periods are different from drowsy periods. This analysis resulted in the identification of four clusters.

a) Cluster \#1 (alert activity > drowsy activity): indicates early periods (70 ms to $280 \mathrm{~ms}$ ), where activity is concentrated in parietal and central electrode sites. These spatial patterns are likely to be involved in early perceptual and evidence accumulation processes.

b) Cluster \#2 (alert activity > drowsy activity): indicates later periods (470 ms to $720 \mathrm{~ms}$ ), where activity is concentrated in occipital, parietal, posterior electrode sites. These spatial patterns are likely to be involved in central processes of evidence accumulation-related processes alone.

c) Cluster \#3 (drowsy activity > alert activity): indicates early periods (70 ms to $330 \mathrm{~ms}$ ), wherein activity is concentrated in frontal/central electrode sites. These spatial patterns are likely to be involved in early perceptual/sensory encoding processes during the drowsy periods.

d) Cluster \#4 (drowsy activity > alert activity): indicates later periods (380 ms to $680 \mathrm{~ms}$ ), wherein activity is concentrated in left frontal electrode sites. These spatial patterns are likely to be involved in central evidence accumulation-related processes alone.

These analyses indicate spatial/temporal regions where the alert and drowsy periods differ in terms of sensory encoding and central evidence accumulation related processes. Furthermore, we have also teased apart these differences from motor related processes by directly using a parameter (drift rate) from the hierarchical drift diffusion modelling.

\section{Arousal modulates frontoparietal cortical neural patterns in perceptual decision making}

Next we performed an exploratory analysis to identify the putative neural sources of these differences in electrodes (Figure 7), we used a source reconstruction procedure to project the classifier patterns of the different clusters back to their neural sources separately (see methods section). In the source space again we performed cluster permutation tests to identify where in the cortex the activity in alert periods is higher than in drowsy periods. We used the actual value of the source activity (instead of absolute values), as we are interested in the distance between the two different patterns and not in overall activity levels.

a) Cluster \#1: These regions are putative locations for early sensory, evidence accumulation related processes in alert periods. The analysis reveals regions (inferior frontal, superior temporal, orbitofrontal) previously associated with the ventral attention network $(32,33)$ in both hemispheres, with emphasis on temporal cortices, in agreement with auditory processing of information. 
b) Cluster \#2: These regions are putative locations for central evidence accumulation related processes in alert periods. The analysis reveals regions (inferior parietal, middle temporal, right lateral prefrontal) that are part of the dorsal attention network $(32,33)$ and central processing of flexible information (34).

c) Cluster \#3: These regions are putative locations for early sensory related processes in drowsy periods. The analysis reveals regions (transverse temporal, superior temporal gyrus,

To summarise, these results indicate that during alert periods: the early sensory/central accumulation related process (until 300ms from stimulus presentation) are located in the regions corresponding to the ventral attention network, further during later periods (after 300ms) evidence accumulation related process are located in the regions corresponding to dorsal attention network (which is specialised for spatial attention). For the drowsy periods: the early sensory encoding related process (until 300ms from stimulus presentation) are located in the regions corresponding to the fronto-parietal, ventral attention network, further during later periods (after 300ms) evidence accumulation related process are located in the regions corresponding to fronto-parietal, dorsal attention network. This indicates that fronto-parietal regions of the brain are further recruited for the late decision-making processes implemented in the drowsy periods, pointing to a cortical 
bioRxiv preprint doi: https://doi.org/10.1101/2020.07.23.218727; this version posted January 23, 2021. The copyright holder for this preprint (which was not certified by peer review) is the author/funder, who has granted bioRxiv a license to display the preprint in perpetuity. It is made available under aCC-BY-NC-ND 4.0 International license.

\section{Projection of regression patterns in source space}

A

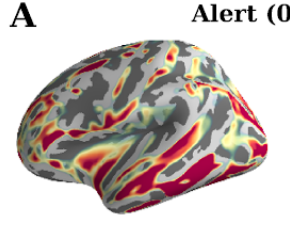

Alert (0.07 - $0.28 \mathrm{~s})$

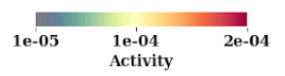

B

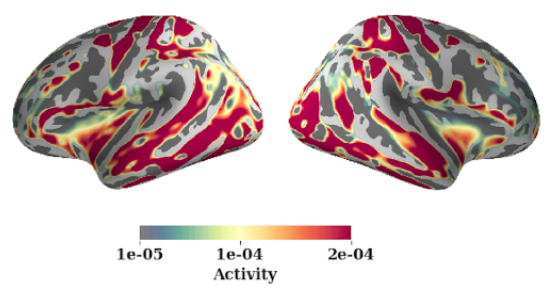

554
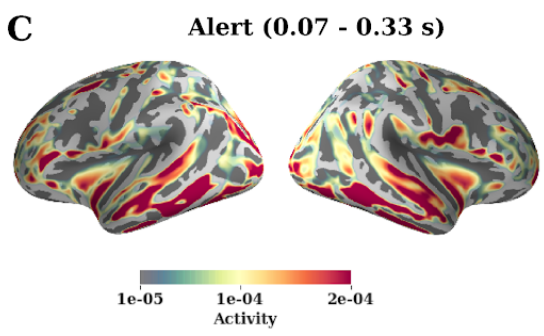

D

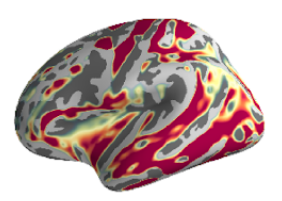

Alert (0.38 - 0.68 s)

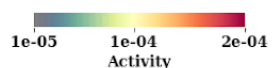

Drowsy (0.07 - $0.28 \mathrm{~s})$
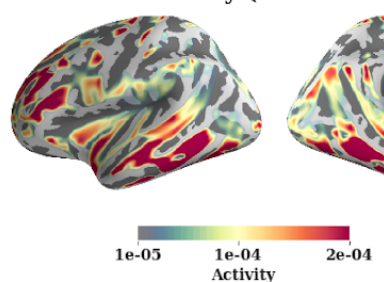

Drowsy (0.47 - 0.72 s)
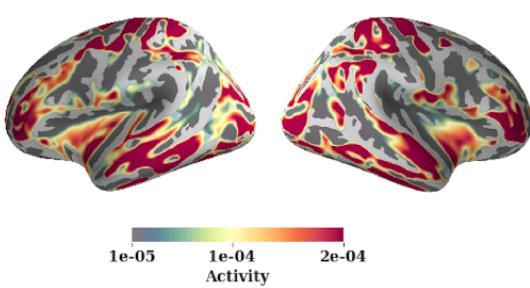

Drowsy (0.07 - 0.33 s)
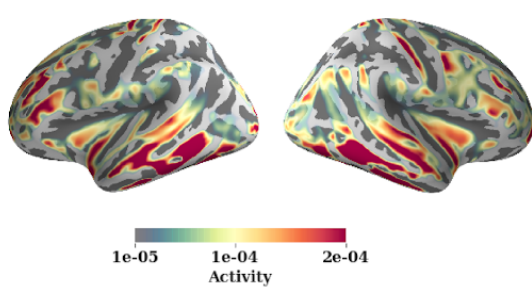

Drowsy (0.38 - 0.68 s)
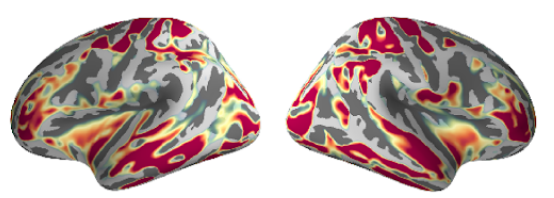

Alert > Drowsy : Cluster\# 1 (0.07 - 0.28 s)
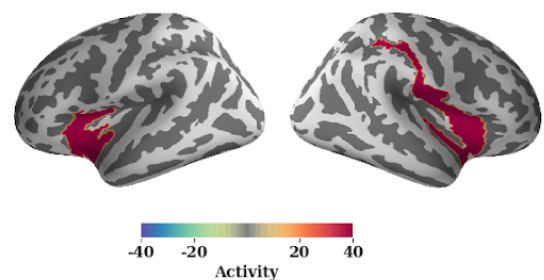

Alert > Drowsy : Cluster\#2 (0.47 - 0.72 s)
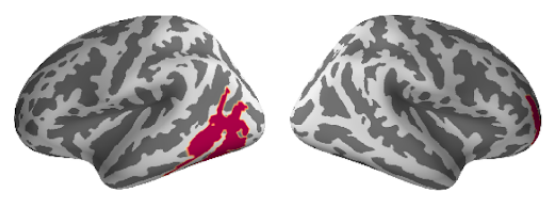

Drowsy > Alert : Cluster\#3 (0.07 - 0.33 s)
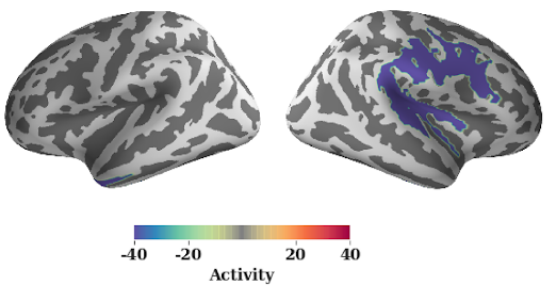

Drowsy > Alert : Cluster\#3 (0.38 - 0.68 s)
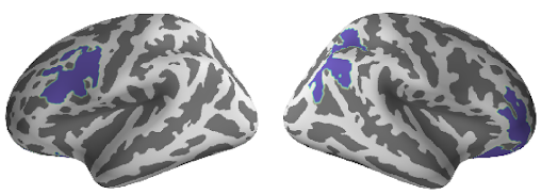

$-40 \quad-20 \quad 20 \quad 40$

555 Figure 8: Regression patterns projected into source space: To identify the putative regions/networks in the brain related to perceptual decision-making and its modulation by alertness we projected the regression patterns in Fig 6 to source space. A) regression patterns in cluster\#1 (Fig 7A) in the early time period in electrode space are projected to source space and spatio-temporal clustering identifies differences in regions related to the ventral attention network. B) similarly regression patterns in cluster\#2 (Fig 7B) reveals differences in dorsal attention networks. C) similarly regression patterns in cluster\#3 (Fig 7C) reveals differences in fronto-parietal regions. D) similarly regression patterns in cluster\#4 (Fig 7D) reveals differences in dorsal attention network and fronto-parietal regions. 


\section{DISCUSSION}

571

In this study we were interested in how changes of arousal modulate perceptual decision making and spatial attention. Informed by previous studies $(25,26)$ we conducted a series of data-driven and model-derived analyses to test different aspects of perceptual and cognitive processes underlying behavioural and neural mechanisms of decision making.

We investigated how alertness modulates different neurocognitive components of perceptual decision-making using a tone localisation task. We replicated the spatial bias induced by alertness changes that has been shown in healthy participants, not dissimilar to that seen following brain damage (unilateral spatial neglect). We characterised behavioural changes in decision making as impacted by the fluctuations in arousal, revealing a decrease in performance due to lower sensitivity to the stimuli but not due to response bias. We also showed a specific effect of alertness reducing the rate of evidence accumulation in a drift diffusion model with little effects on the starting point of the decision (bias). At the neural level we showed that the formation of brain patterns (relating to stimuli presented) is delayed and decreased by lower levels of alertness, suggesting a reconfiguration of the neural networks of the decision. We confirmed this suspicion by mapping the behavioural model parameters of evidence accumulation (drift) into the neural evoked responses, unveiling a more distributed neural patterns, in space and time, in drowsy evidence accumulation and further recruitment of parietal and frontal cortices in low alertness as compared to awake. We think this is evidence for a neural reconfiguration implementing compensatory mechanisms at play in the decision dynamics when challenged by low alertness. For spatial attention theories we are able to add further support to the interaction between ventral attention system (arousal related) and dorsal attention system (spatial attention) when arousal is modulated, and to tease apart the change in the predisposition of the system (bias-point, prior) from the modifications to the decision process itself (drift-rate). For decision making theories, we have shown that low alertness in humans can be used to study evidence accumulation mechanisms and added a new level of description by characterising possible mechanisms of resilience of the neurocognitive processes when tonic alertness decreases. Thus, we were able to show the behavioural flexibility of the system and capture the temporal and spatial neural progression and reconfiguration caused by the change in the internal context - describing its cognitive homeostasis. 


\section{Behavioural changes in decision making show specific modulations of spatial 602 attention with decreased arousal}

603 We established, using multi-level modelling, that the errors for left-sided stimuli increased as 604 participants became drowsy. However the same did not occur for stimuli coming from the right side. 605 This evidence is convergent with Bareham et al(25) but with a different study design, analysis 606 methods and participant sample, furthering the conclusion that there is increased inattention or 607 neglect if stimuli comes from the left side when healthy people become drowsy. Advancing the 608 previous behavioural results, we fitted the proportion of rightward responses of individual 609 participants and identified the subjective midline (radial distance where a participant is equally 610 likely to press left or right response), finding that it shifted to the left side (indicating more right 611 responses) as individuals became drowsy (low alertness). This is in agreement with line-bisection 612 studies(35) showing healthy individuals bisect lines to the left of the veridical midline, 613 overestimating the right side of space. Additional studies(36) have also identified possible neural 614 origins of such bias ('pseudoneglect') and identify task independent activity in the ventral attention 615 network as one of the main modulating factors behind this shift. However these studies did not 616 examine whether trial to trial alertness of individuals was modulated. Several studies $(33,37)$ have 617 shown that activity in the ventral attention network interacts with the arousal system (tonic 618 alertness), and alertness instability, as shown directly in this study, could be a cause for these 619 effects.

\section{Slower evidence accumulation when alertness decreases}

621 To characterise more general aspects of decision making modulated by alertness, we decided to 622 parse the different elements of the process. Using signal detection theory to identify the changes in 623 sensory signal and noise distributions due to alertness, we found that sensitivity (d') was reliably 624 -decreased- by alertness whereas criterion (response bias) was not(38). This suggests that the 625 sensory/perceptual representations have indeed been modulated by alertness, and that participants 626 were not arbitrarily pressing more rightward responses (in the face of uncertain stimuli) as alertness 627 decreases. To complement signal detection theory and further characterise how arousal modulates 628 decision making, we used the rich variability in reaction times produced by awake and drowsy trials 629 in a drift diffusion model, under the assumption that in each trial, noisy evidence is slowly 630 accumulated over time until criterion to make a decision is reached(28). We constructed several 631 models that allowed independent variation of parameters, such as starting point (bias) and drift rate 632 (evidence accumulation), based on stimulus type and alertness levels of the participant. The 633 statistical model with the higher evidence showed that drift rate was modulated by both alertness 634 levels and stimulus type, whereas the starting point was only affected by stimulus type (left or right 635 tone). Further analysis of the winning model also revealed that the evidence for modulation of the 636 starting point was weak while that for drift rate was stronger and reliably different for stimuli from 637 the left side compared to the right side. This shows that alertness indeed differentially modulates 638 evidence coming from the left side of space compared to the right side. Decision making studies in 
639 the visual modality(39) have shown that drift rates are closely related to the strength of stimulus 640 encoding, partially affected by attentional strength, and central evidence accumulation to a 641 decision threshold. Further studies have also shown neural correlates of such parameters in the 642 EEG $(40,41)$ and its relationship with stimuli strength. Hence we consider the effect of alertness 643 modulation on drift rate as acting specifically on spatial attention affecting the stimulus encoding, 644 as well as in central processes of evidence accumulation, revealing a general mechanism where 645 partially independent systems, arousal and decision making, interact.

\section{Alertness delays the neural signatures of perceptual decision making and alters the 648 dynamics of the neural networks}

649 We probed the neural signatures of decision making processes using time-multivariate decoding 650 analysis in high-density EEG. The primary aim was to understand, unconstrained and model-free, 651 how the brain decodes information related to correct decisions when the stimuli presented was 'left' 652 or 'right' under alert and drowsy conditions. Under alert conditions the decoding started early, at $653160 \mathrm{~ms}$ and lasted until $730 \mathrm{~ms}$, however, under drowsy conditions the effects seemed delayed to $654420 \mathrm{~ms}$ and lasted until $730 \mathrm{~ms}$. This $\sim 260 \mathrm{~ms}$ delay may indicate that, under lower arousal, the 655 brain requires a longer time to decipher the direction of stimuli as suggested by slower reaction 656 times and a change in the drift (evidence accumulation) of the diffusion model, or alternatively, that 657 the neural code in the early window of processing is not captured by decoding evoked potentials 658 locked to the stimulus presentation. Furthermore, the overall strength of decoding of the alert 659 condition was systematically higher than the drowsy condition, suggesting that the brain processes 660 responsible for decision making are less variable under the alert condition compared to the drowsy 661 condition, or it may indicate less efficient decision processing during low alertness (see(42) for an 662 account on sleep deprivation on cognition).

663 From a cognitive dynamics perspective the different neural time clusters point potentially to a first 664 stage of information encoding and second of central evidence accumulation (43) that others have 665 mapped to perceptual and central decision processes (3). If that is the case, the first neural signal 666 cluster in drowsy (cluster\#3 in Figure 4C), showing changes in the spatial pattern and a lack of 667 reliable decoding may be interpreted as less efficient encoding and transfer of information in low 668 alertness or may be using different network dynamics that are not captured by the direct decoding. 669 The second cluster (cluster\#4 in Figure 4D) more similar between states, may signal a lower 670 efficiency of central evidence accumulation, as it happens around the time the central decision 671 process occurs in cognitive control(13). Furthermore, the second window of processing may also 672 show part of the motor implementation processes following the evidence accumulation triggering 673 the motor plans, at least for the awake condition (cluster\#2 in Figure 4B), where the responses 674 overlap for several participants (Figure 3D). The overlapping processes from $\sim 400 \mathrm{~ms}$ in awake, a 675 mixture of central evidence accumulation and motor implementation of the decision, depending on 
676 the participant, are easily dissociated when drowsy, when the motor plan is implemented $\sim 500 \mathrm{~ms}$

677 later.

678 Next, we aimed to understand how such neural signatures are depicted at the sensor level. For this, 679 we identified classifier patterns, which are neurophysiologically interpretable(31) from classifier 680 weights. In the alert condition, the early pattern (180-220 ms) indicates activity over frontocentral 681 electrodes, further shifting to more central and parietal sites later on. In the drowsy condition, the 682 pattern initially starts at frontal sites (though not strong enough to be decoded) and shifts to central 683 and parietal channels, later on, from $380 \mathrm{~ms}$ onwards. This indicates that the formation of central, 684 parietal patterns (possibly related to the first stage of encoding, evidence accumulation) takes 685 longer or starts later in drowsy periods compared to alert. Alternatively, the information decoded 686 from the multivariate ERP patterns can be captured in awake but in drowsy the information changes 687 code and it is not captured anymore in this early period. The spatial and temporal distribution of the 688 decoding pattern resembles both a classic P2 or early P3, which has been dubbed as the build-to-threshold signal(44), and the centro-parietal positivity (CPP), a more specific signal of evidence accumulation $(41,45)$ respectively. O'Connell et al showed that the CPP (an EEG evoked response) tracked evidence accumulation in perceptual decision making and was sensitive to perturbation, opening the window for the tracking of signals with sufficient temporal resolution to map the dynamics of decision making in humans(41) beyond what we have learnt in monkeys(14).

694 Following this line of thought, our results highlight the neural dynamics of decision making when 695 the external world remains unaltered, physical evidence held constant, but the internal milieu fluctuates exerting a modulatory influence on the cognitive decision making systems that caused them to reconfigure to solve the task at hand.

\section{Evidence accumulation reveals slower and spatially extended frontoparietal network 699 to resolve the decision when challenged by arousal}

700 The decoding analysis is not guided by parameters that directly test mechanistic hypotheses. It is only based on correct responses from participants and hence it can't reveal the neural source of inattention to the left side of space, or differentiate between cognitive processes proposed by theory. further, the overlap of response periods in the later time window in alert trials, motivates us

704 to use a parameter that can guide neural exploration with a direct readout of evidence accumulation

705 of the decision, avoiding biases and motor related neural signatures. Here we developed a novel

706 method using regression patterns that identified differences in drift rate across alert and drowsy

707 periods into the neural dynamics.

708 We performed a cluster permutation test to identify regions in the brain where temporospatial 709 regression patterns where alert and drowsy periods differ. The spatial topography of these four 710 clusters resemble the clusters from the direct decoding results, providing strength to our 711 preliminary interpretations from the decoding analysis (in the sensor space). To identify the spatial 
712 location of these patterns we projected the data at the sensor level, and to cortical sources for the 713 four different clusters to see the cortical involvement.

714 We first look at clusters(\#1,2) to understand how alert periods are active under this 715 tone-localisation task. These showed regions in the inferior frontal, superior temporal, orbito 716 frontal source regions that resemble the patterns of the ventral attention network with further 717 activity of auditory processing. Although we cannot directly differentiate between early perceptual 718 encoding and central accumulation with this decision, this first cluster most likely corresponds to 719 both in the alert periods. This part of the process of the spatial decision shows the early 720 involvement of these structures that have been linked, in several studies $(46,47)$, to lesions in the inferior frontal and superior temporal gyrus resulting in egocentric neglect (inattention to one side of space from the viewer centric perspective). Furthermore, lower activity in the ventral attention network was also reported for neglect patients suffering from deficits in arousal (33). This agrees with the higher activity observed in the regression patterns for the alert periods compared to the drowsy periods of lower arousal. The cluster\#2, revealed regions in the inferior parietal, middle temporal, right lateral prefrontal source regions that have been associated to the dorsal attention network $(46,47)$. This cluster most likely corresponds to the central evidence accumulation that is directly involved in evaluation of the spatial attention information. To summarise, under alert periods: when the tone is presented, a more ventral attention network gets recruited first, involved in early sensory encoding and evidence accumulation, followed by the information processed further a more dorsal attention network, where the central evidence accumulation primarily takes

732 place to reach the decision.

733 Next we look at clusters(\#3,4) to understand the neural dynamics of evidence accumulation during 734 drowsy periods. Cluster\#3, revealed regions in the transverse temporal, superior temporal gyrus, 735 temporoparietal junction, fronto-parietal regions (inferior frontal gyrus, middle frontal gyrus) in the 736 right hemisphere that can be considered part of the ventral attention network. Also here several 737 studies $(48,49)$ have reported lesions in the right ventral frontal cortex in patients suffering from 738 both arousal related deficits and neglect to the right side of space. The highlighting of these regions 739 in our study indicates that they are different from the alert periods and their higher activity in the 740 regression patterns points to a potential source of spatial neglect (co-occurring with arousal 741 deficits), which is similar to that observed in patient studies. There is the need to further investigate 742 this possible dissociation in further studies, either with lesion metaanalisis or with virtual lesions in 743 normal volunteers. We think this cluster would correspond to a combination of sensory encoding, 744 evidence accumulation in the drowsy periods highlighting an early large cortical reconfiguration of 745 the evidence accumulation process with arousal decreases. Finally Cluster\#4, revealed regions in the 746 inferior parietal sulcus, superior parietal lobule, fronto-polar regions that may be considered part of 747 the dorsal attention network in both hemispheres ascribed to spatial attention processes. 748 Additionally it also revealed further fronto-parietal regions involvement in the left hemisphere, 749 perhaps recruited to provide additional processing to reach criterion to compensate for arousal 750 related deficits. 
Our account of a spatial neglect-like effect in participants under lower arousal is as follows: When participants are fully alert, the information is processed initially by the ventral attention network, followed by the dorsal attention network (specialised in spatial attention). Whereas when participants are drowsy, the ventral attention network although disproportionately affected in the right hemisphere $(33,48,49)$, it is still involved in sensory encoding and early evidence accumulation processes. This reconfiguration in the ventral attention network is further propagated in the next stage, the dorsal attention network. This second part of the decision closely tallies with the proposal by Corbetta and colleagues to account for spatial neglect found in stroke patients suffering from lesions in right-hemispheric regions. But further, this network shows temporal and spatially extended recruitment of the parietal and frontopolar cortices to compensate for the direct effects of arousal in the resolution of the noisy evidence presented to reach a decision in the spatial task.

From a decision making perspective, we find that moving from fully alert to a lower arousal state the brain tries to recruit and expand evidence accumulation related process to fronto-parietal regions instead of the usual temporoparietal regions, consistent with an increase in cognitive demand proposed by the Multiple Demand system (50). Another line of evidence suggesting neural fluctuations influencing evidence accumulation comes from Summerfield and Wyart (51) where the phase of ongoing parietal delta oscillations impacted choice. We interpret that the neural reconfiguration occurring in the transition to lower states of consciousness (inattentive, mildly drowsy) brings slower rhythms and suppresses fast oscillatory events (disappearing alpha and beta), and that this changes the nature of the noise in the brain, forcing the cognitive system to exchange information differently. In this case the arousal changes may be impacting negatively the efficiency of the central accumulation of evidence and the system compensates by extending the neural processes in time and space.

As we lose consciousness, the neural system responsible for decision making adapts to the internal challenge of decreasing arousal and shows its resilience, exerting homeostatic regulations at the cognitive level to maintain performance. The attention and wakefulness fluctuations experienced in humans (and animals) are common during the day and they are not only dependent on the circadian and sleep-wake regulation pressure (52), but also on genetic, epigenetic, environmental and life history factors that shape the alertness aspects of attention as well as the alertness aspect of arousal $(53,54)$.

\section{Concluding remarks}

In this study we investigated the effects of arousal fluctuations on decision making and proposed that despite the internally generated pressure due to the conscious state change, the decision making system is flexible enough to maintain performance by reallocating neural resources. We found that the decision becomes more liberal (in terms of performance) as alertness decreases, showing lower sensitivity with a less steep psychophysics curve. We further show that an evidence accumulation parameter (drift rate) explains the change in the patterns of responses with decreased alertness. At the neural level we demonstrate a change in the manner of processing of the decision 
both by direct decoding of the neural dynamics, and when guided by the evidence accumulation behaviour in each trial.

791

792

\section{Stimuli and Protocol} mechanisms of brain function.

Transitions of consciousness in the near-awake to light-decrease of alertness is emerging as a model for internally caused interactions $(18,26,27,55,56)$. With lesion and pharmacological challenges, neuropsychology and cognitive neuroscience have tried to define the necessary and sufficient brain networks, areas and dynamics of the brain to implement cognition, revealing compensation, reconfiguration and plasticity (57-59). Semi-causal effects of the internal interference exerted by the relative independence of the arousal system on the cognitive process, paves the way for the use of wakefulness and arousal challenges in a principled manner, adding new tools for cognitive brain research. Cognitive neuroscience uses models, correlational and causal methods to reach consensus about the underlying mechanism of thought (60). Here, we have followed a theoretically motivated question about perceptual decision making systems, using behavioural modelling to understand the system, but at the same time directly modulated the neural networks with arousal to uncover the

\section{EXPERIMENTAL PROCEDURES}

Forty-one healthy participants (no auditory, neurological or psychiatric abnormalities) participated in this study. Data from nine participants was discarded due to a) technical problems with headphone amplifier (8) b) Not following task instructions (1). Thus only data from 32 participants (24.46 \pm 3.72 years old, 14 males) was considered for further analysis. All participants were self-reported to be right-handed and the same was also established by using Edinburgh Handedness Scale (61). Each participant had a handedness score of above 0 (right-handed) with mean $80.26 \pm$ 23.59. Only easy sleepers (as per self report) were recruited and further they were administered with the Epworth Sleepiness scale (62) on the day of the experiment. 29 participants had a sleepiness score $>=7$ (classified as easy sleepers) and 3 of them had a sleepiness score $>=4$. All participants were asked not to consume any stimulant like Coffee/Tea before the experiment that day. The study and the experimental protocol was approved by the cambridge psychology research ethics committee and written informed consent was provided by all participants. A monetary compensation of $£ 30$ was provided for participation in the study.

Each participant underwent two experimental sessions a) Alert b) Drowsy.

Alert session: participants were presented with 124 complex harmonic tones (guitar chord) that fell on the left or right side of their veridical midline $\left(0^{\circ}\right)$ ranging from $-59.31^{\circ}$ to $+59.31^{\circ}$. These tones were recorded using in-ear microphones in free-field (25). Six tones from $-59.31^{\circ}$ to $-39.26^{\circ}$ were 
827 presented two times each; twelve tones from $-35.24^{\circ}$ to $-1.86^{\circ}$ were presented four times each. A 828 similar pattern was repeated on the right side with twelve tones from $1.86^{\circ}$ to $35.24^{\circ}$ presented four times each, six tones from $39.26^{\circ}$ to $59.31^{\circ}$ presented two times each. The tones in the midline $\left(0^{\circ}\right)$ were presented four times, resulting in a total of 124 tones. The order of tones presented was randomized per participant. Further, participants were instructed to keep their eyes closed and respond (as quickly and as accurately as possible) with a button press (using left/right thumb) indicating the location of the tone (left or right). Each trial began after a random interval of 2-3 seconds and if the participant did not respond for 5 seconds, the next trial was started. The participants were also instructed to stay awake throughout this session.

836

Drowsy session: participants were presented with 740 complex harmonic tones (as above) that fell on the left or right of their veridical midline $\left(0^{\circ}\right)$ again ranging from $-59.31^{\circ}$ to $+59.31^{\circ}$. Six tones from $-59.31^{\circ}$ to $-39.26^{\circ}$ were presented twenty times each; twelve tones from $-35.24^{\circ}$ to $-1.86^{\circ}$ were presented twenty times each. Similar pattern was repeated on the right side with twelve tones from $1.86^{\circ}$ to $35.24^{\circ}$ being presented twenty times each, six tones from $39.26^{\circ}$ to $59.31^{\circ}$ presented twenty times each. The tone in the midline $\left(0^{\circ}\right)$ was presented twenty times, resulting in a total of 740 tones. The order of tones was again randomized per participant as in the alert session. participants were again instructed to keep their eyes closed and respond (as quickly and as accurately as possible) with a button press (by left/right thumb) indicating the direction of the tone (left or right). Each trial began after a random interval of 4-5 seconds and if the participant did not respond for 5 seconds, the next trial was started. In this session, the participants were allowed to fall asleep (and become drowsy) and were gently awoken if they didn't respond to more than 3 trials consecutively.

850 Before the start of the experiment, the participants were allowed a practise session to familiarise 851 with the task.

\section{Preprocessing}

854 EEG data was acquired with $129 \mathrm{Ag} / \mathrm{AgCl}$ electrodes (Electrical Geodesics Inc) using $\mathrm{Cz}$ as the 855 reference electrode. The impedances of all electrodes were kept below $100 \mathrm{~K} \Omega$ (to ensure higher 856 signal to noise ratio) and data was acquired at a sampling rate of $500 \mathrm{~Hz}$. EEG data was 857 pre-processed with custom made scripts in MATLAB (MathWorks Inc. Natick, MA, USA) using 858 EEGLAB toolbox (63). The preprocessing steps are as follows: First, the peripheral electrodes that 859 covered the regions of forehead, cheeks and neck were removed to reduce artifacts related to eye 860 and muscle movements, thus retaining only 92 channels that covered the scalp. Second, the data 861 was bandpass filtered with zero phase shift between 1 and $40 \mathrm{~Hz}$ using hamming windowed-sinc FIR 862 filter and further resampled to $250 \mathrm{~Hz}$. Third, pre-trial and post-trial epochs per trial were created. 863 For the pre-trial epochs, the data was epoched from $-4000 \mathrm{~ms}$ to $0 \mathrm{~ms}$ prior to the onset of the 864 stimuli. The pre-trial epochs were created only in the drowsy session and not in the alert session 865 (details below). For the post-trial epochs, the data was epoched from -200ms to 800ms to the onset 866 of the stimuli for both the alert and drowsy sessions. Fourth, the trials that exceeded the amplitude 
867 threshold of $\pm 250 \mu \mathrm{V}$ were removed in a semi-automatic fashion. Fifth, the bad channels were 868 detected in a two-step fashion: a) channels are considered bad (zero activity) if channel variance is below 0.5 . b) The normalized power spectrum of the remaining channels was computed and any channel that exceeded the mean power spectrum by \pm 3 standard deviations was marked bad. Sixth, to remove further artifacts related to eye-blink and muscle movement, independent component analysis (ICA) was performed on the channels not marked as bad in the previous step. ICA components that correspond to artifacts were rejected by manual inspection. Seventh, the bad channels were now interpolated using spherical interpolation. Eighth, the bad trials were detected again using an amplitude threshold of $\pm 250 \mu \mathrm{V}$ and bad electrodes (those exceeding the threshold) in such trials were interpolated in a trial-by-trial fashion. Ninth, the post-trial epochs were re-referenced to the average of all channels (whereas the pre-trial epochs were maintained with the

878 recorded reference $-\mathrm{Cz}$ ).

\section{Alertness levels}

The preliminary step in both behavioural and neural analysis is to classify periods of data into 'alert' and 'drowsy'. The data from the pre-trial epochs were used to classify each trial into alert or drowsy. For the alert session, all pre-trial periods were considered to be awake as participants were explicitly instructed to stay awake (and none of the participants failed to respond to any of the trials in the alert session). In the drowsy session, the participants were allowed to fall asleep and hence some trials would be considered as alert and some would be drowsy. For each trial in the drowsy session, pre-trial epochs were analysed using the micro-measures algorithm (24). Each trial was classed as 'alert', 'drowsy(mild)' , 'drowsy(severe)'. Further only trials that were classified as 'drowsy(mild)' were used as drowsy trials. The other trials were excluded because usually participants don't respond when under 'drowsy(severe)' trials. We similarly excluded the 'alert' trials in the drowsy session to instead compare the drowsy trials of the drowsy session to all of the trials in the alert 892 session.

\section{Behavioral analysis}

\section{Error proportion}

897 In order to understand how the rate of errors differs across different stimuli (tones to the left or 898 right side of midline) and how it is modulated by change in alertness levels we performed the 899 following analysis. First, we computed the proportion of errors made by each participant under each 900 alertness level ('awake', 'drowsy') and under each stimulus type ('left' or 'right' tone). If the total 901 number of trials for any participant under any condition is less than 5, then the corresponding error 902 proportion (for that condition) is ignored in the further analysis. We defined 4 different multilevel 903 models to understand the modulation of error proportion by state of participant ('alert', 'drowsy') 904 and stimulus ('left', 'right'). We used multilevel models, as different participants had different 905 levels of alertness (differing number of alert, drowsy trials). In the null model, the error proportion 906 depends only on the mean per participant (fixed effect) and the participant id (random effect). In 
907 the second model (state model), the error proportion depends only on the state of the participant 908 ('alert' or 'drowsy' as fixed effect) and individual participant (participant id is used as random 909 effect). In the third model (stimulus model), the error proportion depends only on the stimulus 910 ('left' or 'right' as fixed effect) and individual participant (participant id is used as random effect). In 911 the fourth model (state-stimulus model), the error proportion depends on a combination of state of 912 participant ('alert' or 'drowsy') and the stimulus ('left' or 'right'), both used as fixed effects and 913 individual participants (participant id is used as random effect). These 4 models were fitted using 914 the 'Imer' function ('lmerTest' package) in R (64) and the winning model is identified as the one 915 with the highest log-likelihood by comparing it with the null model and performing a likelihood 916 ratio chi-square test $(\chi 2)$. Finally the top two winning models were compared against each other 917 using 'anova' function in R (65), to validate whether the winning model (if it is more complex) is 918 actually better than the losing model (if it is simpler). The state-stimulus model emerged as the 919 winning model.

921 The different models along with their log-likelihood values are shown below:

925 Model comparison:

926

\begin{tabular}{|l|l|l|l|}
\hline Model & Parameters & Log-likelihood & $\operatorname{Pr}(>\boldsymbol{\chi 2} \mathbf{2})$ \\
\hline Null & Fixed: mean, Random: participant id & 61.24 & - \\
\hline State & Fixed: state, Random: participant id & 66.62 & $<0.001$ \\
\hline Stimulus & Fixed: stimulus, Random: participant id & 68.45 & $<0.001$ \\
\hline State-Stimulus & Fixed: state*stimulus, Random: participant id & $\mathbf{7 7 . 9 8}$ & $<0.001$ \\
\hline
\end{tabular}

927

928 Type III Analysis of Variance Table with Satterthwaite's method of the winning model 929 (State-Stimulus):

930

\begin{tabular}{|l|l|l|l|l|l|l|}
\hline Model elements & Sum Sq & Mean Sq & NumDF & DenDF & F value & $\operatorname{Pr}(>$ F) \\
\hline State & 0.21023 & 0.21023 & 1 & 95.07 & 14.0465 & 0.0003061 \\
\hline Stimulus & 0.28140 & 0.28140 & 1 & 95.07 & 18.8018 & $3.607 \mathrm{e}-05$ \\
\hline State:Stimulus & 0.10312 & 0.10312 & 1 & 95.07 & 6.8897 & 0.0101019 \\
\hline
\end{tabular}

932 The state-stimulus (winning model) was further analysed with the 'anova' function and it was found 933 that there was a reliable main effect of both state $(\mathrm{p}<0.001)$ and stimulus $(\mathrm{p}<0.0001)$ on error 934 proportion and also the interaction between state and stimulus also had a reliable effect $(p<0.05)$ on 
935 error proportion. Estimated marginal means were computed using the 'emmeans' package in R to 936 perform post-hoc tests using tukey adjustment for multiple comparisons.

\section{Subjective midline shifts}

939 The change in subjective midline was quantified by fitting psychometric functions to the responses produced by each participant under alert and drowsy conditions.

The proportion of rightward responses for each participant under each stimulus condition from $-60^{\circ}$ 943 to $+60^{\circ}$ was fitted with a cumulative normal function using the generalized linear model 'glm' 944 function in R. The link function used in the model was 'probit' which quickly asymptotes to the 945 axes compared to the 'logit' function. The fit also constrained in such a way the number of trials 946 under each stimulus angle weighed the shape of the function. The mean of such a cumulative normal function (the point where the curve crosses 0.5 in the y-axis) is referred to as the subjective midline ('bias'). The subjective midline is the stimulus (angle) at which the participant performs at chance $(0.5)$, which in an ideal world would be closer to the veridical midline $\left(0^{\circ}\right)$. This fitting procedure was performed for each participant separately under each condition (alert, drowsy). Examples of fits for two participants are shown in the supplemental information (Figure s1). The standard deviation of the cumulative function (slope or steepness) represents the 'sensitivity' of the 953 system. In general, large variations in the bias point tend to reduce the sensitivity of the system.

954 Further we also ignored participants (leaving 11 participants) in the drowsy condition that had a 955 bias point of more than $60^{\circ}$ (as the overall stimulus angle can vary only between $-60^{\circ}$ to $+60^{\circ}$ ) and 956 standard deviation of more than $30^{\circ}$.

\section{Drift-diffusion modelling}

960 The different elements of the decision-making process can be teased apart by using the drift-diffusion model. The primary parameters of this model include

962 i) drift-rate - ' $v$ ' -- rate of evidence accumulation

963 ii) bias point - ' $z$ ' -- starting point of the decision making process

964 iii) boundary separation distance - 'a' -- distance between the two decision boundaries

965 iv) non-decision time -'Ter' -- for accounting other processes like stimulus encoding (before the 966 start of evidence accumulation process), response execution (after the end of evidence 967 accumulation).

969 We implemented the drift diffusion process using hierarchical drift diffusion model (HDDM) (66) 970 (version 0.7.1) that allows for a hierarchical Bayesian procedure to estimate the model parameters. 971 The principal reason for using such hierarchical procedures is because different participants fall 972 asleep in different ways (differing number of alert and drowsy trials). Using a hierarchical bayesian 973 procedure allows for robust estimation of model parameters under such limited conditions of trials 974 available (67). We used the response of each participant ('left' or 'right' button press) instead of 
975 accuracy ('correct' or 'incorrect') to fit the HDDM. Such a procedure is referred to as 976 Stimulus-coding and allows for robust estimation of ' $z$ ' without being biased by accuracy. To 977 identify the best parameters, we identified 8 different models where each model allows the 978 parameters ('z','v') to vary depending on state ('alert' or 'drowsy') or stimulus ('left' or 'right'). In 979 each model, 15000 samples from the posterior distribution were estimated using Markov chain

\section{Neural analysis}

\section{5}

\section{Decoding}

997 We used multivariate pattern analysis (MVPA) techniques to analyse the divergent pattern in EEG data. Decoding is a form of MVPA in which we analyse patterns of brain activity in order to predict 999 the experimental condition (or stimuli) under which that pattern was generated. Traditional ERP 1000 (Event related potentials) analyses rely on using a-priori identified spatial locations or temporal 1001 segments in the data to measure the differences across conditions. However decoding techniques do 1002 not rely on a-priori definitions and perform much better in detecting differences across 1003 experimental conditions (30). 
1005 Temporal decoding:

1006 Temporal decoding involves using EEG data $(X)$ composed of size: [Electrodes $\mathrm{x}$ Time points $\mathrm{x}$ 1007 Trials] to predict the experimental condition $(Y)$. The experimental condition predicted here was 1008 the direction of the stimuli presented e.g. left or right stimuli. The first step in the decoding analysis 1009 consists of fitting an estimator $(w)$ to a subset of the data $(X)$ called (Xtrain) to predict a subset of 1010 the experimental condition $(Y)$ called (Ytrain). The second step involves using this trained 1011 estimator on another subset of the data $(X)$ called (Xtest) to predict subset of the experimental 1012 condition $(Y)$ called ( $Y$ test $)$. The third step involves evaluating the performance of this estimator 1013 using a measure (e.g. accuracy) by comparing the prediction ( $\hat{Y}$ test) with the actual label (Y test).

1014

1015 Estimator construction:

1016 First, the EEG data is subjected to a standard scaler (using StandardScaler() from scikit-learn version 1017 0.22) that removes the mean of the data and scales it by its variance. Second, we used logistic 1018 regression to estimate the model parameters for maximally separating the different experimental 1019 conditions. Third, we implemented temporal decoding by using the sliding estimator (using 1020 SlidingEstimator() from scikit-learn) to fit the logistic regression model per time-point.

1021

1022 Cross-validation:

1023 The EEG data was first downsampled to $100 \mathrm{~Hz}$ and further binary classification was performed 1024 between two conditions ('left' and 'right' stimuli) separately across alert and drowsy conditions per 1025 individual participant. As the target of the classification was stimuli being presented we only 1026 considered the trials wherein the participant made the correct decision. The participant was 1027 considered for classification only if they had at least 25 trials under each condition. Further 5-fold 1028 cross validation was performed such that 4 folds were used for training and the last fold was used as 1029 a testing set.

1030

1031 Validation measure:

1032 The classifier performance was evaluated using Area Under the Curve (AUC) of the 1033 receiver-operating characteristic (ROC). It is implemented using 'roc_auc' in the sliding estimator 1034 function in scikit-learn. When AUC is about 0.5 the classifier performs at chance, while the AUC 1035 score of 1 has a very good separability across classes. We computed the AUC-ROC score per 1036 participant as the average of the score across all the cross-validation folds.

1037

1038 Group statistics:

1039 In order to identify the reliability of the AUC score at the group level, we performed a cluster 1040 permutation test (participants $\mathrm{x}$ timepoints) using MNE version 0.19.2 (spatio_temporal_ 1041 cluster_1samp_test) (70). Thus producing p-values per time point at the group level, from which 1042 time points wherein we can infer those regions wherein AUC is reliably different from chance (0.5) 1043 at the group level. 


\section{Coefficients of patterns:}

1045 The parameters of the decoding model are not neurophysiologically interpretable in a 1046 straightforward way (31). Hence the parameters of the backward model (decoding) need to be 1047 transformed to produce the forward model. This is done by obtaining the coefficients of the 1048 estimator model per participant using the 'get_coef' function from MNE('_patterns'). For performing 1049 group statistics in electrode space, we used the same cluster permutation based approach as 1050 described earlier.

1051

\section{Source reconstruction of patterns:}

1053 The coefficients created above can be projected into the source space to infer the brain regions 1054 involved in the pattern activity. Source reconstruction was achieved using a combination of 1055 Freesurfer (71) and MNE. First, the surface was reconstructed using 'recon-all' (Freesurfer) using the 1056 default ICBM152 template for the structural magnetic resonance image (MRIs). Next, the Boundary 1057 element model (BEM) was created using 'make_watershed_bem' from MNE. Further, scalp surfaces 1058 for the different element boundaries were created using 'make_scalp_surface' from MNE. Second, we 1059 performed the registration of the scalp surface with the default EEG channel locations (with 1060 fiducials as markers) manually using 'coregistration' from MNE. Third, forward solution was 1061 computed using 'make_bem_model' from MNE with conductivity $=[0.3,0.006,0.3]$. Fourth, to test if 1062 the source reconstruction of the electrode data is accurate we projected the ERP data of a sample 1063 participant into source space and analysed data from different regions of interest to confirm its 1064 validity as shown in the supplemental information (Figure s4). The classifier patterns of each 1065 participant were then projected into source space in the following manner. First, we computed the 1066 noise covariance using the baseline data from -0.2 to $0 \mathrm{~ms}$. Second, we used the forward solution 1067 (8196 vertices) and the noise covariance to create an inverse operator using 1068 'minimum_norm.make_inverse_operator' from MNE (loose $=0.2$ ). Third, used the individual 1069 classifier pattern per participant and applied the inverse operator on it (method $=\mathrm{MNE}, \mathrm{SNR}=3$, 1070 lambda $\left.2=1 /(\mathrm{SNR})^{2}\right)$ to produce the source reconstruction of the classifier patterns per participant.

1071 For performing group statistics in the source space we used the same cluster permutation based 1072 approach as described earlier.

1073

\section{Decoding locked to responses of participants:}

1075 To tease apart the process related to evidence accumulation from motor implementation, we 1076 decided to decode the response hand of the participant. The EEG data for the response decoding was 1077 created by epoching the data from $-800 \mathrm{~ms}$ to $200 \mathrm{~ms}$ prior to the onset of the response from the 1078 participant. The response-locked trials were preprocessed in a similar fashion to the 1079 stimulus-locked trials (details in the section of Preprocessing). The alertness level for the 1080 corresponding response-locked trial was obtained from the labels of the stimulus-locked trial. The 1081 temporal decoding and other methods used are similar to the stimulus-locked trials, except that the 1082 target of decoding is the response hand being pressed (left or right thumb). 


\section{Neuro-behavioural analysis}

1085

\section{Regression with drift diffusion model}

1087 To identify the correlates of the evidence accumulation process, we used the model parameters 1088 generated by the drift diffusion model (winning model \#8). First, the ERP data (post trial epochs) 1089 were z-scored per electrode per trial. Second, the ERP data was baseline corrected with pre-trial 1090 data from $-200 \mathrm{~ms}$ to $0 \mathrm{~ms}$. Third, the ERP data was averaged every 50ms per electrode per trial to 1091 create 20 time points (-200ms to $800 \mathrm{~ms}$ ) per electrode per trial. Fourth, the ERP data were entered 1092 into a regression with trial by trial estimation of the drift rate using the HDDMRegressor from the 1093 HDDM toolbox (66). The drift rate was allowed to vary per trial based on the ERP data per state 1094 ('alert' or 'drowsy') per stimuli ('left' or 'right'). Fifth, the traces were computed per condition (state 1095 and stimuli combination). Sixth, the differences in drift rate (between left and right stimuli) per 1096 time point per electrode were computed in both alert and drowsy condition. This difference 1097 represents the discriminability of the electrode in identifying the left and right stimuli at that time 1098 point. Thus this analysis yielded differences in drift rate per electrode, per time point, per 1099 condition, per participant. The differences can then be considered similar to the classifier patterns 1100 and can be analysed both in electrode and source spaces. Further we also computed group level 1101 differences using the cluster permutation techniques described earlier.

1102

\section{Reproducing figures}

1104 The code for reproducing the main figures, raw datasets and other data accompanying this paper 1105 will further be released upon acceptance or during the review of the paper.

\section{AUTHOR CONTRIBUTIONS}

1109 Conceptualization: S.R.J and T.A.B.

1110 Methodology: S.R.J.

1111 Software: S.R.J.

1112 Investigation: S.R.J.

1113 Formal Analysis: S.R.J.

1114 Resources: S.R.J, C.A.B. and T.A.B.

1115 Data Curation: S.R.J and C.A.B.

1116 Writing - Original Draft: S.R.J.

1117 Writing - Review \& Editing: S.R.J, C.A.B, and T.A.B.

1118 Visualization: S.R.J.

1119 Supervision: C.A.B. and T.A.B.

1120 Project Administration: S.R.J.

1121 Funding Acquisition: S.R.J and T.A.B. 


\section{ACKNOWLEDGMENTS}

1124

1125 This research was funded by the Gates Cambridge Scholarship awarded to S.R.J and the Wellcome

1126 Trust Biomedical Research Fellowship WT093811MA awarded to T.A.B. as well as small

1127 departmental funding to S.R.J. and T.A.B. We thank Annamaria Laudini and Dritan Nikolla for

1128 assistance with data collection, Andrés Canales-Johnson, Will Harrison, Valdas Noreika and other

1129 members of the Consciousness and Cognition Lab in Cambridge for their valuable comments and

1130 support. We also thank lavazza for the continuous support with delicious and stimulating coffee.

\section{SUPPLEMENTAL INFORMATION}

\section{Different stages of a cognitive task}

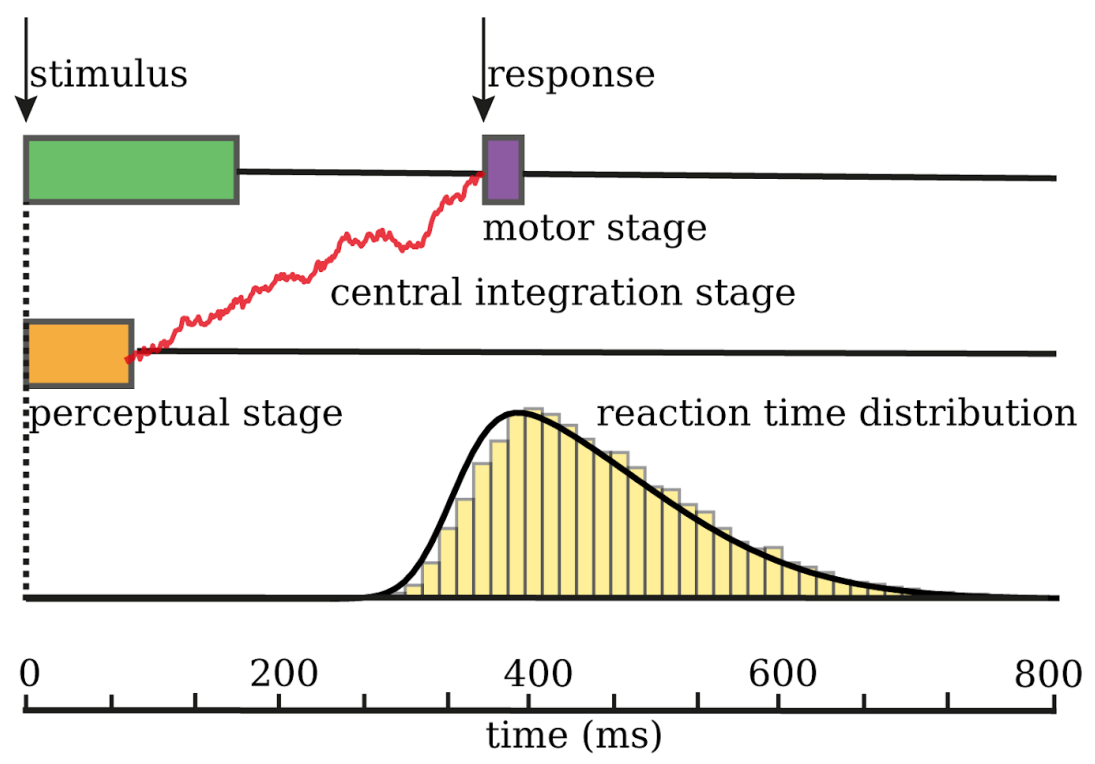

1136 Figure s1: Different stages of a cognitive task as identified by (2). In this example task, the stimulus period is 1137 represented by a green box and lasts from 0 till $180 \mathrm{~ms}$ and response occurs at approximately $400 \mathrm{~ms}$. The 1138 different stages of processing are: a) perceptual stage (orange box) representing encoding of stimuli to 1139 decision making areas of the brain. b) central integration stage (red line) that represents accumulation of 1140 evidence towards a decision threshold. c) motor stage (violet box) represents the implementation of the 1141 decision from the previous stage. The distribution of reaction times for this example task are also plotted as 1142 histograms (yellow). 


\section{Subjective midline by sigmoid fits}

$\mathbf{A}$

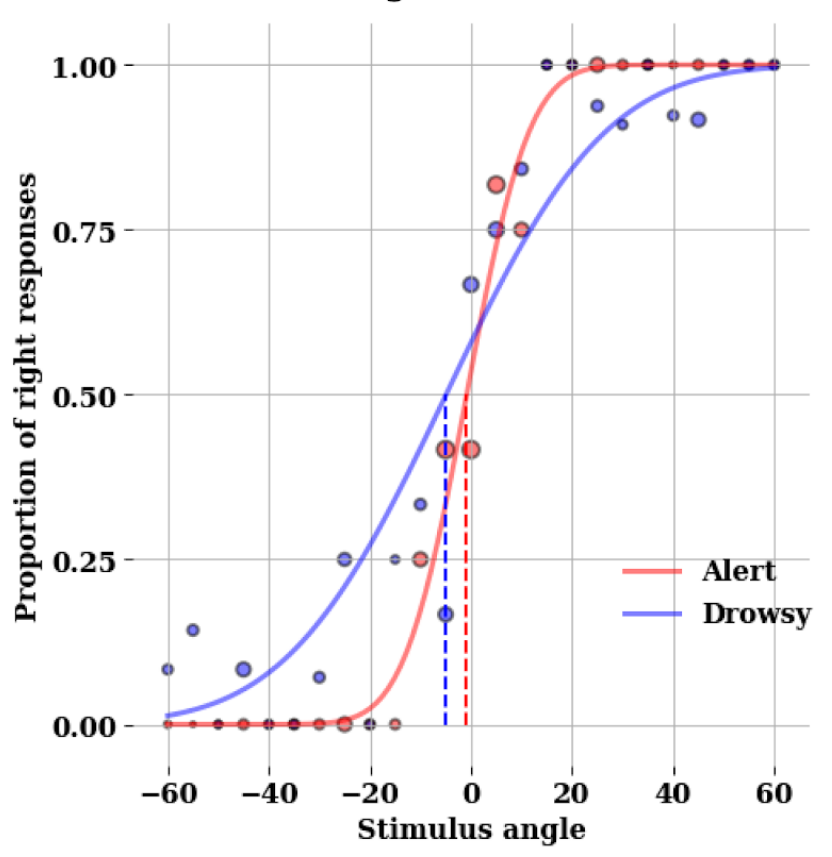

B midline shifting towards right side

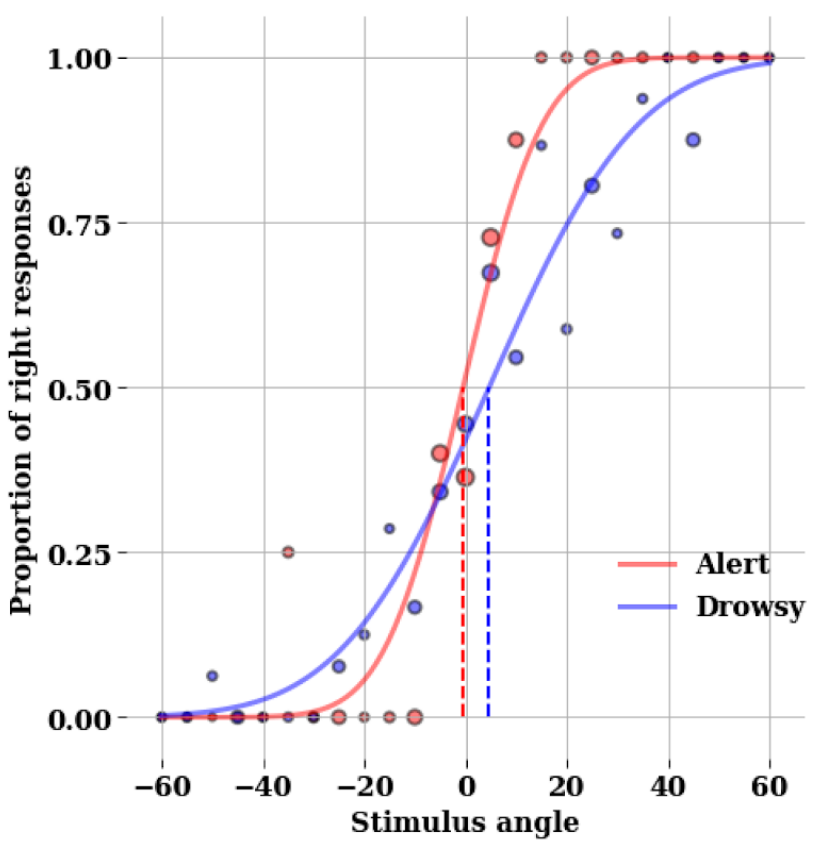

1146 Figure s2: Example fits of two different participants are shown. For each participant under each condition

1147 ('alert', 'drowsy'), the proportion of rightward responses were fitted to the stimulus angle varying from $-60^{\circ}$

1148 to $+60^{\circ}$. The 'glm' fit was constrained in such a way that the number of responses under each angle was used

1149 as weights. The size of the dots represent the normalised (per condition) number of trials under each angle. A)

1150 bias point (dotted line) shifts towards the left side as the participant becomes drowsy. In other words, as the

1151 participant becomes drowsy they overestimate the right side of space. B) bias point (dotted line) shifts

1152 towards the right side as the participant becomes drowsy. In other words, as the participant becomes drowsy

1153 they overestimate the left side of space 


\section{Posterior predictive fits from the best model}

\section{Posterior predictive distributions from the best model}

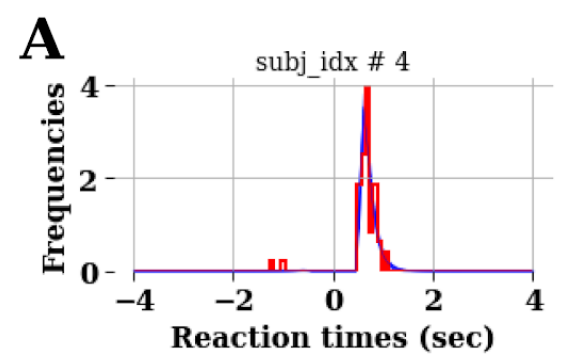

B

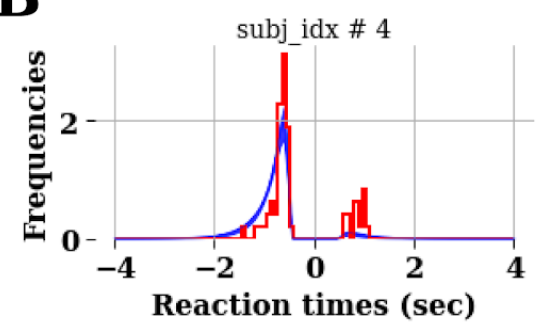

1166
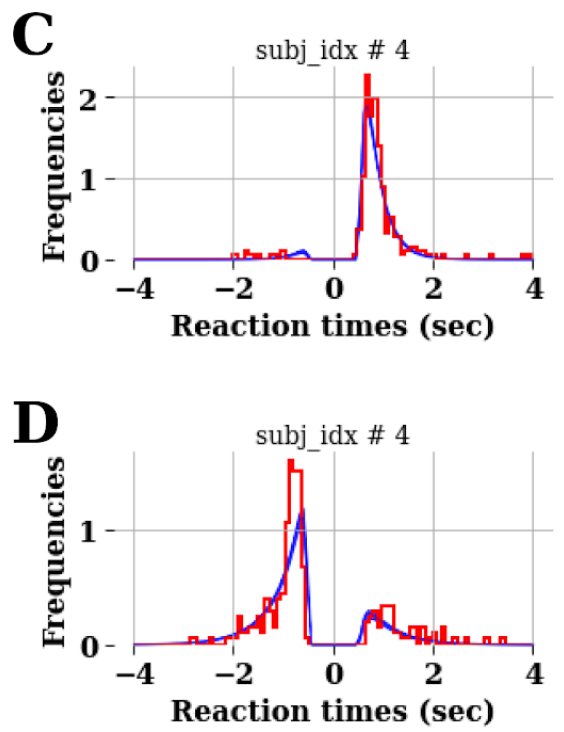

State: Alert -- Stimulus: Left
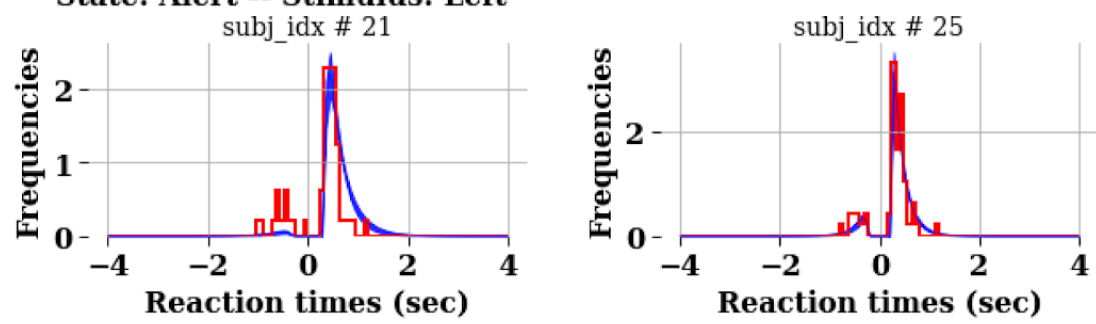

State: Alert -- Stimulus: Right subj_idx \# 21
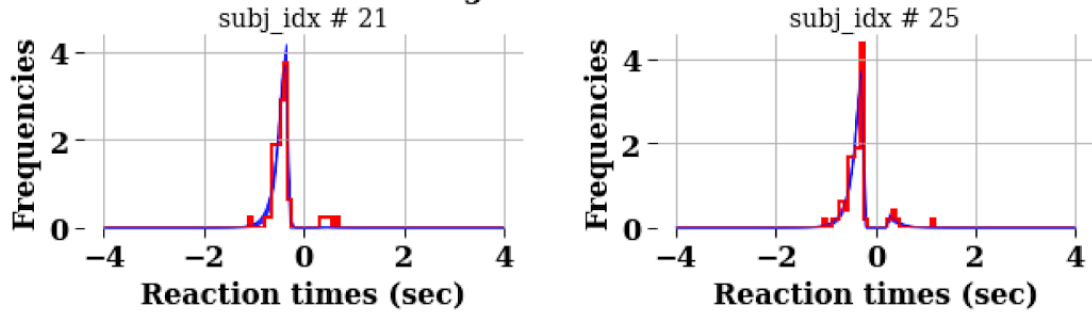

State: Drowsy -- Stimulus: Left
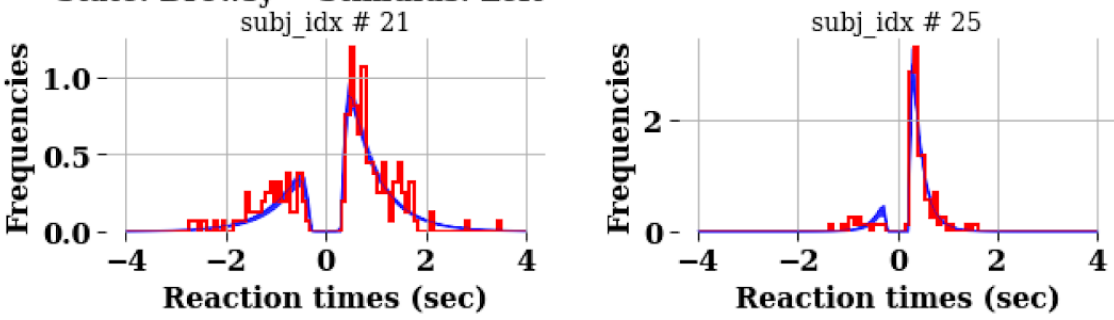

State: Drowsy -- Stimulus: Right
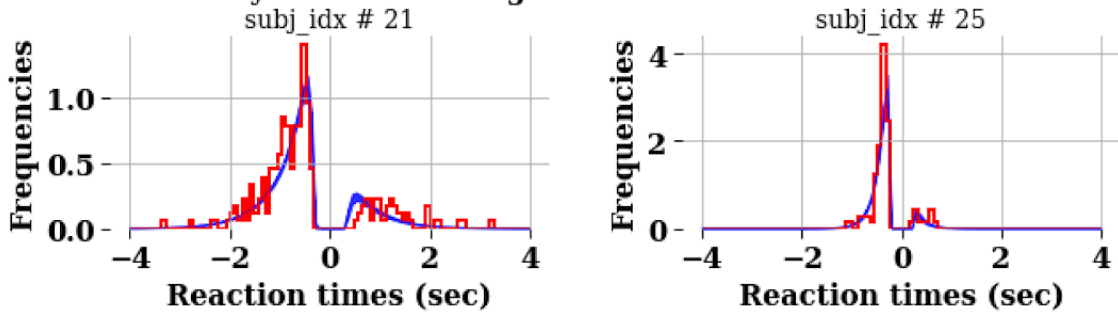

1167 Figure s3: Posterior predictive fits for the best model (\#8) in hddm for some sample participants are shown. 1168 The $\mathrm{x}$-axis in each plot represents the reaction times in seconds, the positive values are times for a 'left' 1169 button presses, whereas those on the negative x-axis are for 'right' button presses. The histograms (in red) 1170 represent distributions of the observed data, whereas the blue lines represent model prediction. A) Posterior

1171 predictive distributions when the participant is 'alert' and the stimulus presented was 'left' tone. B) Posterior

1172 predictive distributions when the participant is 'alert' and the stimulus presented was 'right' tone. C)

1173 Posterior predictive distributions when the participant is 'drowsy' and the stimulus presented was 'left' tone. 
1174 D) Posterior predictive distributions when the participant is 'drowsy' and the stimulus presented was 'right'

1175 tone.

1176

1177 Event related potential (ERP) values at significant electrodes from classifier patterns

ERP values at electrodes idenfied from clustering of classifier patterns

A

Alert > Drowsy : Cluster\# 1 (0.16 - 0.32 s)

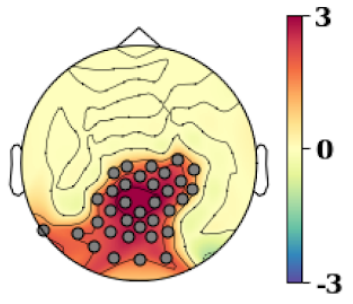

F-stat (mean)

B

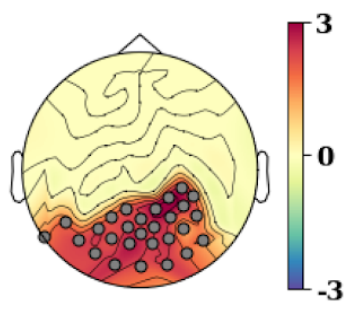

F-stat (mean)

C

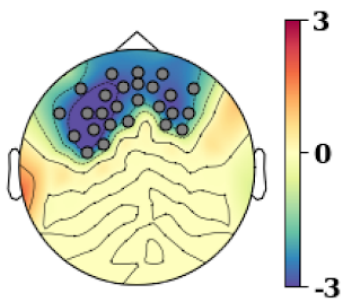

F-stat (mean)

D

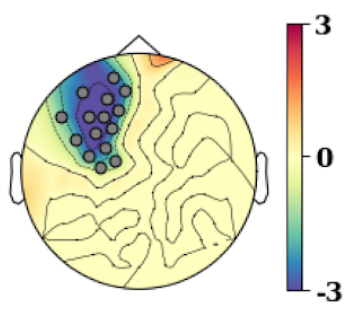

F-stat (mean)

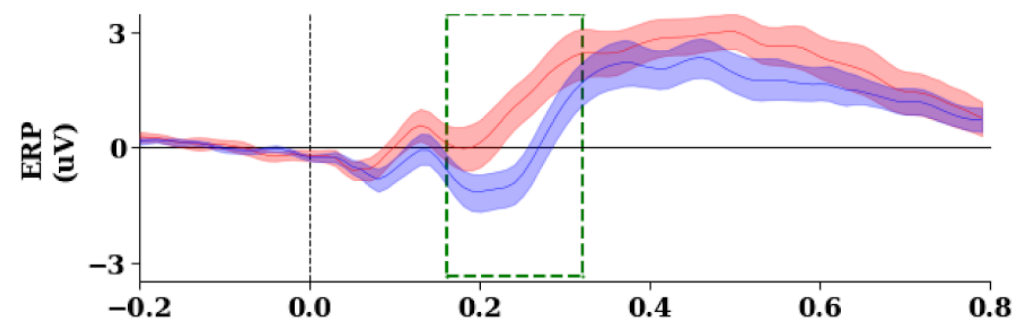

Time (s)

Alert > Drowsy : Cluster\#2 (0.44 - 0.62 s)

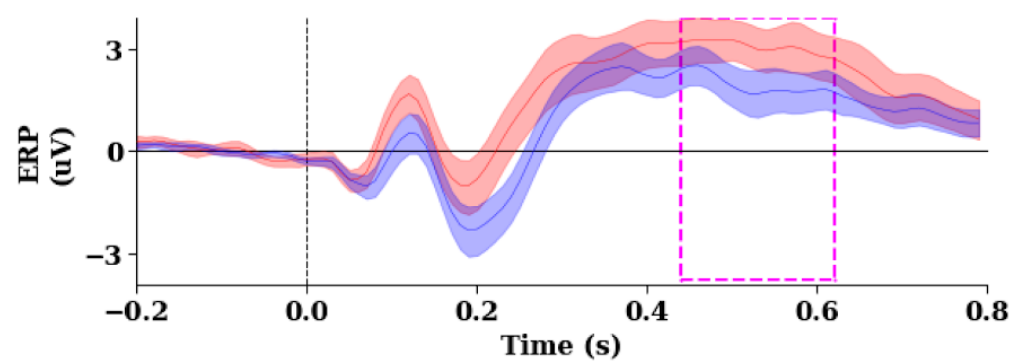

Drowsy > Alert : Cluster\#3 (0.18 - 0.31 s)

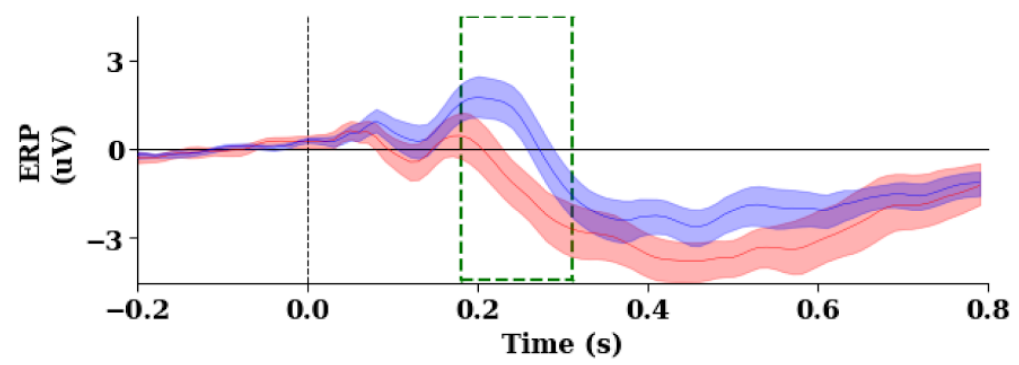

Drowsy $>$ Alert : Cluster\#4 (0.39 - 0.62 s)

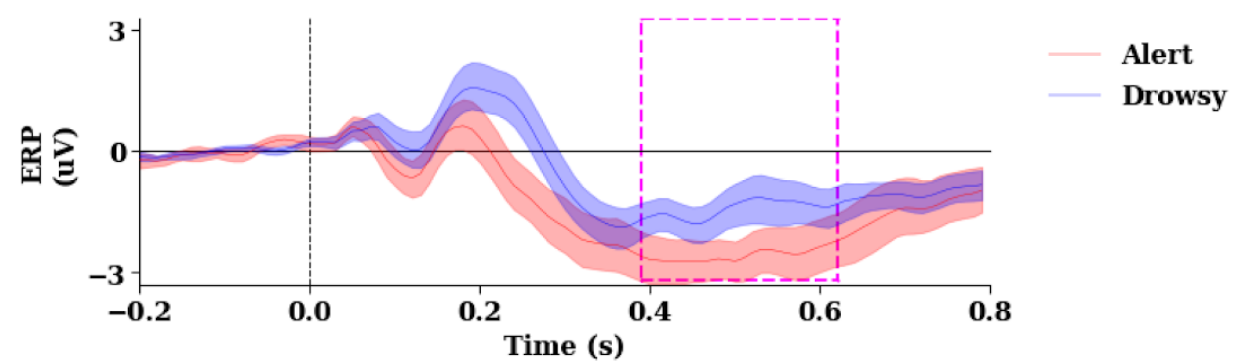

Alert Drowsy

Alert Drowsy

lert 
1179 Figure s4: spatio-temporal clustering of the classifier patterns across alert and drowsy periods reveals

1180 significant clusters at different time periods (from Figure 4). Here the significant electrodes are taken and

1181 their corresponding ERP values are plotted. The time course of the ERP values look similar to the time course 1182 of the classifier patterns itself.

1183

\section{Classifier patterns for temporal decoding of responses}

A

Alert

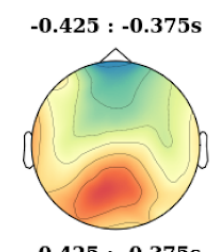

$-0.425:-0.375 s$

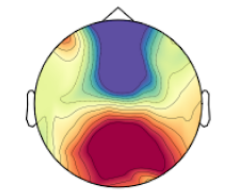

B

Alert

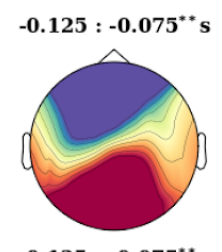

ons

Drowsy

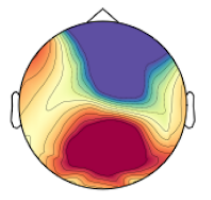

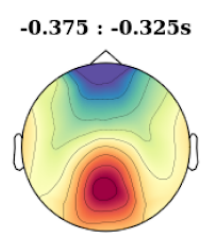

$-0.375:-0.325 s$
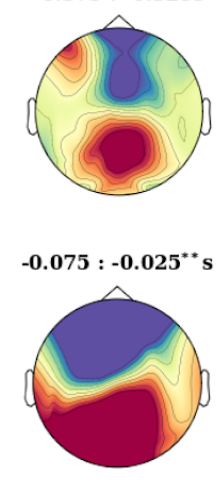

$-0.075:-0.025^{* *} s$

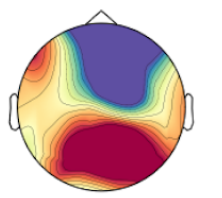

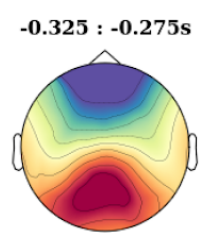

$-0.325:-0.275 s$
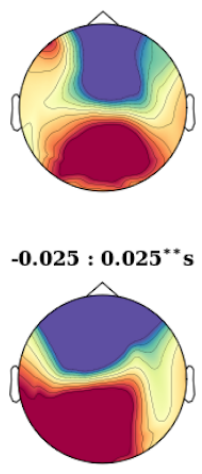

$-0.025: 0.025 * s$

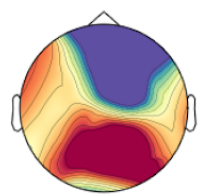

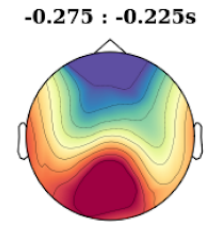

$-0.275:-0.225 s$
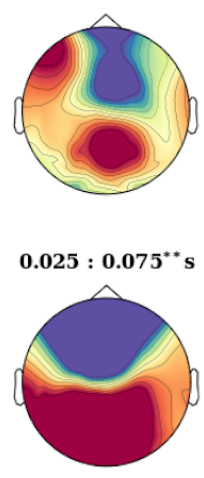

$0.025: 0.075^{* *} s$

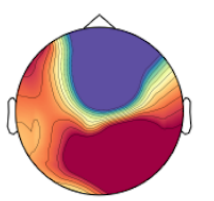

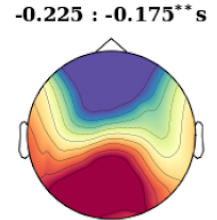

$-0.225:-0.175 s$
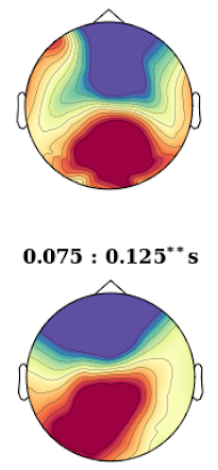

$0.075: 0.125^{* *} s$

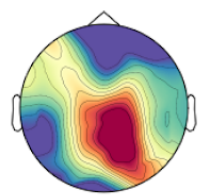

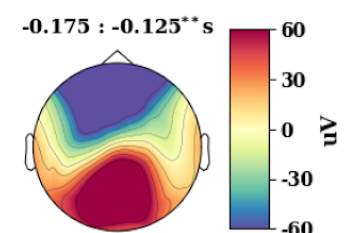

$-0.175:-0.125^{* *} s \quad 30$
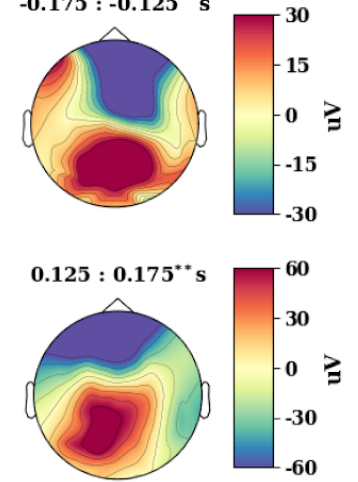

$0.125: 0.175^{* *} s \quad T^{30}$

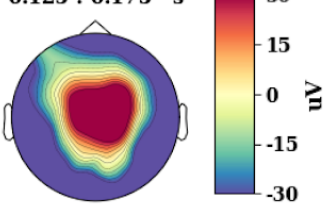

Figure s5: Temporal decoding (responses): A,B) Comparison of coefficients of classifier patterns in alert and drowsy periods. The patterns look fairly similar except the changes in intensity levels. The amplitude of the patterns are also several levels of magnitude higher (alert: $60 \mathrm{uV}$, drowsy: $30 \mathrm{uV}$ ) than temporal decoding 1185 of stimuli (both alert, drowsy: $2.4 \mathrm{uV}$ Fig 3F,G).

1187 The classifier patterns for alert when response-locked (Figure s5), reveals scalp patterns related to 1188 motor implementation that seem similar to the later period of decoding when stimulus-locked ( 0.3 1189 onwards in Alert in Figure $3 F, G),(-0.175$ to $-0.125 \mathrm{sec}$ in Alert in Figure s5B). One possible 1190 interpretation is that these neural patterns may represent the same processes from 0.3 sec onwards 1191 in Figure 3F, motor implementation, as seen in the decoding for the response-locked. However, the 1192 classifier patterns for drowsy (Figure s5B) related to motor implementation, despite being spatial 1193 similar to stimulus-related decoding (0.42 to $0.46 \mathrm{sec}$ in Drowsy in Figure 3G), (-0.175 to $-0.125 \mathrm{sec}$ 1194 in Drowsy in Figure s5B), these are separated by $300-700 \mathrm{~ms}$, with $0.42 \mathrm{sec}$ drowsy decoding to 1195 actual reaction times (mean) only occurring at $1.17 \mathrm{sec}$. Hence, the decoding (of stimulus) under 
1196 drowsy conditions seems to start later, and this process is clearly different from motor related 1197 processes and shows an evidence accumulation related pattern that most likely develops later in a 1198 motor related process. This is different from the alert condition, wherein the classifier performance 1199 was initially driven by perceptual and central evidence accumulation processes (from 0.18 sec to 0.3 $1200 \mathrm{sec}$ ) and further by motor implementation related processes, possibly from $0.3 \mathrm{sec}$ onwards.

\section{Validation of source reconstruction}

1201

1202 To verify the accuracy of the spatial localization procedure, we used the following methodology. 1203 First, we used EEG data from a sample participant (in the drowsy periods, as it has a larger number 1204 of trials) and computed noise covariance from $0.2 \mathrm{sec}$ to $0 \mathrm{sec}$ (pre stimulus period) using the 1205 'shrunk' method in mne. Next the computed covariance was regularised. Second, the forward 1206 solution was computed using the transformation file, source space, beamformer solution as 1207 described in the methods section. Third, we computed the inverse operator using the noise 1208 covariance and the forward solution. Fourth, we applied the inverse operator on the raw data with 1209 parameters of $\mathrm{snr}=12$, lambda2 $=1.0 / \mathrm{snr}^{2}$, method $=$ 'MNE'. For validation we used the 'MNE' 1210 method instead of 'dSPM' used in the decoding analysis, as we wanted to have a positive and 1211 negative amplitude in the source signals. Further we only picked the source signals whose 1212 orientations were normal to the surface. Fifth, we used labels from the left hemisphere in the source 1213 space and extracted signals in the regions of auditory, occipital, middle temporal, and frontal pole 1214 using the mode 'mean_flip'. Sixth, we extracted mean time courses inside each ROI and plotted the 1215 same in Figure s3. We identified N100 (peak negative signal $100 \mathrm{~ms}$ followed by stimulus 1216 presentation) only in the time course from the auditory region, whereas the other ROIs displayed 1217 weaker N100 amplitudes as shown below. This validates the accuracy of source localization as N100 1218 is ERP amplitude is known to be localised in the auditory region.

1219

\section{source localization in left hemisphere rois in a sample participant}

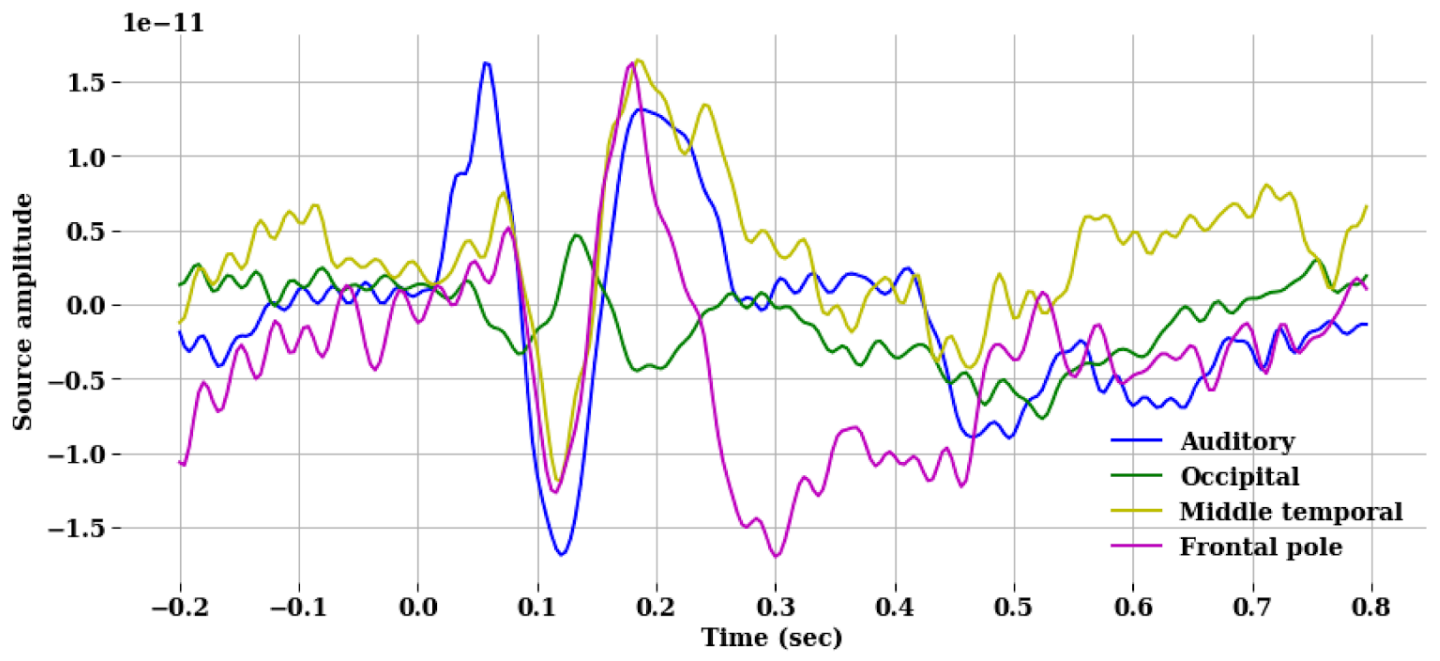


1221 Figure s6: source localization performed in a sample participant clearly reveals N100 presence in auditory

1222 regions at $100 \mathrm{~ms}$ after stimulus presentation. However occipital regions do not reveal the presence of N100

1223 thereby validating the source localization methods used.

1224

\section{REFERENCES}

1226 1. Buchanan L, O’Connell A. A brief history of decision making. Harv Bus Rev. 2006

1227 Jan;84(1):32-41, 132.

1228 2. Sigman M, Dehaene S. Parsing a cognitive task: a characterization of the mind's bottleneck.

1229 PLoS Biol. 2005 Feb;3(2):e37.

1230 3. Sigman M, Dehaene S. Brain mechanisms of serial and parallel processing during dual-task 1231 performance. J Neurosci. 2008 Jul 23;28(30):7585-98.

1232 4. O’Connell RG, Shadlen MN, Wong-Lin K, Kelly SP. Bridging Neural and Computational

1233 Viewpoints on Perceptual Decision-Making. Trends Neurosci. 2018 Nov;41(11):838-52.

1234 5. Hull JT, Wright KP Jr, Czeisler CA. The influence of subjective alertness and motivation on 1235 human performance independent of circadian and homeostatic regulation. J Biol Rhythms. 2003 1236 Aug;18(4):329-38.

1237 6. Knowles JB. Sleep, Sleepiness and Performance Timothy H. Monk (Ed.) John Wiley \& Sons, 1991 1238 [Internet]. Vol. 14, Journal of Organizational Behavior. 1993. p. 703-5. Available from:

1239 http://dx.doi.org/10.1002/job.4030140710

1240 7. Goupil L, Bekinschtein TA. Cognitive processing during the transition to sleep. Arch Ital Biol. 2012 Jun;150(2-3):140-54.

1242 8. Link SW, Heath RA. A sequential theory of psychological discrimination. Psychometrika. 1975 1243 Mar;40(1):77-105.

1244 9. Gold JI, Shadlen MN. The neural basis of decision making. Annu Rev Neurosci. 2007;30:535-74.

1245 10. Heekeren HR, Marrett S, Ungerleider LG. The neural systems that mediate human perceptual 1246 decision making. Nat Rev Neurosci. 2008 Jun;9(6):467-79.

1247 11. Heekeren HR, Marrett S, Bandettini PA, Ungerleider LG. A general mechanism for perceptual 1248 decision-making in the human brain. Nature. 2004 Oct 14;431(7010):859-62.

1249 12. Heekeren HR, Marrett S, Ruff DA, Bandettini PA, Ungerleider LG. Involvement of human left 1250 dorsolateral prefrontal cortex in perceptual decision making is independent of response 1251 modality. Proc Natl Acad Sci U S A. 2006 Jun 27;103(26):10023-8.

1252 13. Ho TC, Brown S, Serences JT. Domain general mechanisms of perceptual decision making in 1253 human cortex. J Neurosci. 2009 Jul 8;29(27):8675-87. 
1254 14. Shadlen MN, Newsome WT. Neural Basis of a Perceptual Decision in the Parietal Cortex (Area LIP) of the Rhesus Monkey [Internet]. Vol. 86, Journal of Neurophysiology. 2001. p. 1916-36. Available from: http://dx.doi.org/10.1152/jn.2001.86.4.1916

1257 15. Shadlen MN, Newsome WT. Motion perception: seeing and deciding. Proc Natl Acad Sci U S A. 1996 Jan 23;93(2):628-33.

1259 16. Roitman JD, Shadlen MN. Response of neurons in the lateral intraparietal area during a combined visual discrimination reaction time task. J Neurosci. 2002 Nov 1;22(21):9475-89.

1261 17. Bekinschtein T, Cologan V, Dahmen B, Golombek D. You are only coming through in waves: wakefulness variability and assessment in patients with impaired consciousness [Internet]. Progress in Brain Research. 2009. p. 171-89. Available from: http://dx.doi.org/10.1016/s0079-6123(09)17712-9

1264 18. Canales-Johnson A, Beerendonk L, Blain S, Kitaoka S, Ezquerro-Nassar A, Nuiten S, et al. Decreased Alertness Reconfigures Cognitive Control Networks. J Neurosci. 2020 Sep 9;40(37):7142-54.

1267 19. McGinley MJ, Vinck M, Reimer J, Batista-Brito R, Zagha E, Cadwell CR, et al. Waking State: Rapid Variations Modulate Neural and Behavioral Responses. Neuron. 2015 Sep $23 ; 87(6): 1143-61$.

1270 20. de Gee JW, Colizoli O, Kloosterman NA, Knapen T, Nieuwenhuis S, Donner TH. Dynamic modulation of decision biases by brainstem arousal systems. Elife [Internet]. 2017 Apr 11;6.

1271 Available from: http://dx.doi.org/10.7554/eLife.23232

1272 21. van Kempen J, Loughnane GM, Newman DP, Kelly SP, Thiele A, O’Connell RG, et al. Behavioural and neural signatures of perceptual decision-making are modulated by pupil-linked arousal. Elife [Internet]. 2019 Mar 18;8. Available from: http://dx.doi.org/10.7554/eLife.42541

1275 22. Wang C-A, Baird T, Huang J, Coutinho JD, Brien DC, Munoz DP. Arousal Effects on Pupil Size, Heart Rate, and Skin Conductance in an Emotional Face Task [Internet]. Vol. 9, Frontiers in Neurology. 2018. Available from: http://dx.doi.org/10.3389/fneur.2018.01029

1278 23. Hori T, Hayashi M, Morikawa T. Topographical EEG changes and the hypnagogic experience [Internet]. Sleep onset: Normal and abnormal processes. 1994. p. 237-53. Available from:

1280 http://dx.doi.org/10.1037/10166-014

1281 24. Jagannathan SR, Ezquerro-Nassar A, Jachs B, Pustovaya OV, Bareham CA, Bekinschtein TA. Tracking wakefulness as it fades: Micro-measures of alertness. Neuroimage. 2018 Aug $1283 \quad 1 ; 176: 138-51$.

1284 25. Bareham CA, Manly T, Pustovaya OV, Scott SK, Bekinschtein TA. Losing the left side of the world: rightward shift in human spatial attention with sleep onset. Sci Rep. 2014 May 28;4:5092.

1287 26. Noreika V, Canales-Johnson A, Johnson A, Arnatkevičiūtė A, Koh J, Chennu S, et al. 
1311 35. Jewell G, McCourt ME. Pseudoneglect: a review and meta-analysis of performance factors in

Wakefulness fluctuations elicit behavioural and neural reconfiguration of awareness [Internet]. 2020 [cited 2020 Sep 7]. p. 155705. Available from: https://www.biorxiv.org/content/10.1101/155705v3

27. Comsa IM, Bekinschtein TA, Chennu S. Transient Topographical Dynamics of the Electroencephalogram Predict Brain Connectivity and Behavioural Responsiveness During Drowsiness. Brain Topogr. 2019 Mar;32(2):315-31.

8. Ratcliff R, Smith PL, Brown SD, McKoon G. Diffusion Decision Model: Current Issues and History. Trends Cogn Sci. 2016 Apr;20(4):260-81.

9. Kruschke JK. Bayesian Estimation Supersedes the t Test [Internet]. PsycEXTRA Dataset. 2012. Available from: http://dx.doi.org/10.1037/e502412013-055

30. Fahrenfort JJ, van Driel J, van Gaal S, Olivers CNL. From ERPs to MVPA Using the Amsterdam Decoding and Modeling Toolbox (ADAM). Front Neurosci. 2018 Jul 3;12:368.

1. Haufe S, Meinecke F, Görgen K, Dähne S, Haynes J-D, Blankertz B, et al. On the interpretation of weight vectors of linear models in multivariate neuroimaging. Neuroimage. 2014 Feb 15;87:96-110.

2. Yeo BTT, Thomas Yeo BT, Krienen FM, Sepulcre J, Sabuncu MR, Lashkari D, et al. The organization of the human cerebral cortex estimated by intrinsic functional connectivity [Internet]. Vol. 106, Journal of Neurophysiology. 2011. p. 1125-65. Available from: http://dx.doi.org/10.1152/jn.00338.2011

33. Corbetta M, Shulman GL. Spatial neglect and attention networks. Annu Rev Neurosci. 2011;34:569-99.

34. Fedorenko E, Duncan J, Kanwisher N. Broad domain generality in focal regions of frontal and parietal cortex. Proc Natl Acad Sci U S A. 2013 Oct 8;110(41):16616-21. line bisection tasks. Neuropsychologia. 2000;38(1):93-110.

36. Benwell CSY, Harvey M, Thut G. On the neural origin of pseudoneglect: EEG-correlates of shifts in line bisection performance with manipulation of line length. Neuroimage. $2014 \mathrm{Feb}$ 1;86:370-80.

37. Robertson IH, Mattingley JB, Rorden C, Driver J. Phasic alerting of neglect patients overcomes their spatial deficit in visual awareness. Nature. 1998 Sep 10;395(6698):169-72.

38. Deaton M, Tobias JS, Wilkinson RT. The Effect of Sleep Deprivation on Signal Detection Parameters [Internet]. Vol. 23, Quarterly Journal of Experimental Psychology. 1971. p. 449-52. Available from: http://dx.doi.org/10.1080/14640747108400257

39. Smith PL, Ratcliff R. An integrated theory of attention and decision making in visual signal 
detection. Psychol Rev. 2009 Apr;116(2):283-317.

1323 40. Nunez MD, Vandekerckhove J, Srinivasan R. How attention influences perceptual decision making: Single-trial EEG correlates of drift-diffusion model parameters. J Math Psychol. 2017 Feb;76(Pt B):117-30.

1326 41. O’Connell RG, Dockree PM, Kelly SP. A supramodal accumulation-to-bound signal that determines perceptual decisions in humans. Nat Neurosci. 2012 Dec;15(12):1729-35.

1328 42. Killgore WDS. Effects of sleep deprivation on cognition. Prog Brain Res. 2010;185:105-29.

1329 43. Kelly SP, O’Connell RG. The neural processes underlying perceptual decision making in humans: Recent progress and future directions. Journal of Physiology-Paris. 2015 Feb

$1331 \quad 1 ; 109(1): 27-37$.

1332 44. Twomey DM, Murphy PR, Kelly SP, O’Connell RG. The classic P300 encodes a build-to-threshold decision variable. Eur J Neurosci. 2015 Jul;42(1):1636-43.

1334 45. Loughnane GM, Newman DP, Bellgrove MA, Lalor EC, Kelly SP, O’Connell RG. Target Selection Signals Influence Perceptual Decisions by Modulating the Onset and Rate of Evidence Accumulation [Internet]. Vol. 26, Current Biology. 2016. p. 496-502. Available from: http://dx.doi.org/10.1016/j.cub.2015.12.049

1338 46. Shulman GL, Pope DLW, Astafiev SV, McAvoy MP, Snyder AZ, Corbetta M. Right hemisphere dominance during spatial selective attention and target detection occurs outside the dorsal 1339 frontoparietal network. J Neurosci. 2010 Mar 10;30(10):3640-51.

1340 47. Dietz MJ, Friston KJ, Mattingley JB, Roepstorff A, Garrido MI. Effective connectivity reveals right-hemisphere dominance in audiospatial perception: implications for models of spatial neglect. J Neurosci. 2014 Apr 2;34(14):5003-11.

1343 48. Heilman KM, Bowers D, Valenstein E, Watson RT. Hemispace and Hemispatial Neglect. In:

1344 Jeannerod M, editor. Advances in Psychology. North-Holland; 1987. p. 115-50.

1345 49. Corbetta M, Kincade MJ, Lewis C, Snyder AZ, Sapir A. Neural basis and recovery of spatial 1346 attention deficits in spatial neglect. Nat Neurosci. 2005 Nov;8(11):1603-10.

1347 50. Duncan J. The multiple-demand (MD) system of the primate brain: mental programs for

1348 intelligent behaviour. Trends Cogn Sci. 2010 Apr;14(4):172-9.

1349 51. Wyart V, de Gardelle V, Scholl J, Summerfield C. Rhythmic fluctuations in evidence

1350 accumulation during decision making in the human brain. Neuron. 2012 Nov 21;76(4):847-58.

1351 52. Borbély AA, Daan S, Wirz-Justice A, Deboer T. The two-process model of sleep regulation: a

1352 reappraisal. J Sleep Res. 2016 Apr;25(2):131-43.

1353 53. Bekinschtein TA, Dehaene S, Rohaut B, Tadel F, Cohen L, Naccache L. Neural signature of the 
1356 54. Mitchell KJ. Innate: How the Wiring of Our Brains Shapes Who We Are. Princeton University

1357 Press; 2020. 312 p.

1358 55. Tagliazucchi E, Laufs H. Decoding wakefulness levels from typical fMRI resting-state data

1359 reveals reliable drifts between wakefulness and sleep. Neuron. 2014 May 7;82(3):695-708.

1360 56. Song C, Tagliazucchi E. Linking the nature and functions of sleep: insights from multimodal imaging of the sleeping brain [Internet]. Vol. 15, Current Opinion in Physiology. 2020. p. 29-36. Available from: http://dx.doi.org/10.1016/j.cophys.2019.11.012

1362 57. Adolphs R. Human Lesion Studies in the 21st Century. Neuron. 2016 Jun 15;90(6):1151-3.

1363 58. Valero-Cabré A, Amengual JL, Stengel C, Pascual-Leone A, Coubard OA. Transcranial magnetic stimulation in basic and clinical neuroscience: A comprehensive review of fundamental principles and novel insights [Internet]. Vol. 83, Neuroscience \& Biobehavioral Reviews. 2017. p. 381-404. Available from: http://dx.doi.org/10.1016/j.neubiorev.2017.10.006

1365 59. Yeung AWK, Tzvetkov NT, Atanasov AG. When Neuroscience Meets Pharmacology: A Neuropharmacology Literature Analysis [Internet]. Vol. 12, Frontiers in Neuroscience. 2018.

1367 Available from: http://dx.doi.org/10.3389/fnins.2018.00852

1368 60. Krakauer JW, Ghazanfar AA, Gomez-Marin A, MacIver MA, Poeppel D. Neuroscience Needs 1369 Behavior: Correcting a Reductionist Bias. Neuron. 2017 Feb 8;93(3):480-90.

1370 61. Oldfield RC. The assessment and analysis of handedness: the Edinburgh inventory. Neuropsychologia. 1971 Mar;9(1):97-113.

1372 62. Johns MW. A new method for measuring daytime sleepiness: the Epworth sleepiness scale. Sleep. 1991 Dec;14(6):540-5.

1373 63. Delorme A, Makeig S. EEGLAB: an open source toolbox for analysis of single-trial EEG dynamics including independent component analysis. J Neurosci Methods. 2004 Mar

1375 15;134(1):9-21.

1376 64. Kuznetsova A, Brockhoff PB, Christensen RHB. lmerTest Package: Tests in Linear Mixed Effects Models [Internet]. Vol. 82, Journal of Statistical Software. 2017. Available from: http://dx.doi.org/10.18637/jss.v082.i13

1379 65. Fox J, Weisberg S. An R Companion to Applied Regression. SAGE Publications; 2018. 608 p.

1380 66. Wiecki TV, Sofer I, Frank MJ. HDDM: Hierarchical Bayesian estimation of the Drift-Diffusion Model in Python. Front Neuroinform. 2013 Aug 2;7:14.

1382 67. Zhang J, Rittman T, Nombela C, Fois A, Coyle-Gilchrist I, Barker RA, et al. Different decision deficits impair response inhibition in progressive supranuclear palsy and Parkinson's disease. 
1385 68. Spiegelhalter DJ, Best NG, Carlin BP, van der Linde A. Bayesian measures of model complexity and fit [Internet]. Vol. 64, Journal of the Royal Statistical Society: Series B (Statistical Methodology). 2002. p. 583-639. Available from: http://dx.doi.org/10.1111/1467-9868.00353

1388 69. Gelman A, Carlin JB, Stern HS, Dunson DB, Vehtari A, Rubin DB. Bayesian Data Analysis, Third Edition. CRC Press; 2013. 675 p.

1390 70. Gramfort A, Luessi M, Larson E, Engemann DA, Strohmeier D, Brodbeck C, et al. MEG and EEG data analysis with MNE-Python. Front Neurosci. 2013 Dec 26;7:267.

1392 71. Fischl B. FreeSurfer. Neuroimage. 2012 Aug 15;62(2):774-81. 